\title{
Geometric integration by parts and Lepage equivalents*
}

\author{
Marcella Palese $^{a}$, Olga Rossi and Fabrizio Zanello ${ }^{b}$ \\ ${ }^{a}$ Department of Mathematics, University of Torino \\ via C. Alberto 10, 10123 Torino, Italy \\ e-mail: MARCELLA.PALESE@UNITO.IT \\ ${ }^{b}$ Institute of Mathematics, University of Göttingen \\ Bunsenstraße 3-5, D-37073 Göttingen, Germany \\ e-mail: FABRIZIO.ZANELLO@MATHEMATIK.UNI-GOETTINGEN.DE
}

\begin{abstract}
We compare the integration by parts of contact forms - leading to the definition of the interior Euler operator - with the so-called canonical splittings of variational morphisms. In particular, we discuss the possibility of a generalization of the first method to contact forms of lower degree. We define a suitable Residual operator for this case and, working out an original conjecture by Olga Rossi, we recover the Krupka-Betounes equivalent for first order field theories. A generalization to the second order case is discussed.
\end{abstract}

Key words: Interior Euler operator; Residual operator; geometric integration by parts; Poincaré-Cartan form; Lepage equivalent.

2010 MSC: 53Z05,58A20,58Z05.

\section{Introduction}

The Euler-Lagrange operator can be geometrically described by means of two interrelated geometric objects (and corresponding geometric integration

* Our colleague and friend Olga Rossi passed away in October 2019. This paper is an outcome of our collaboration, which we miss heartily, and we dedicate it to her memory. 
by parts procedures), the one based on the concept of differential forms and exterior differential modulo contact structures, the other based on the interpretation of variational objects as fibered morphisms [7, 8, 3] etc.

Following an approach inaugurated by the works of Cartan and Lepage, the finite order variational sequence was introduced and developed by Krupka; see e.g. [12. The problem of the representation of the finite order variational sequence (whose objects, we recall, are equivalence classes of local differential forms) has been discussed in terms of the so called interior Euler operator; see e.g. [9] and [16].

On the other hand variational morphisms [3] not only provide a geometric formulation of the calculus of variations, but in general of a wide class of differential operators. Their most relevant property is that, under certain conditions, they admit canonical and algorithmic splittings and, by the introduction of a connection on the base manifold and a connection on the considered fiber bundle, globality and uniqueness properties of these splittings can be assured.

The aim of this paper is to investigate the relation between these two approaches which use different geometric integration by parts techniques.

We perform the identification of contact forms with variational morphisms for 1-contact forms of degree at most $n+1$, where $n$ is the dimension of the base manifold of the considered fiber bundles. The two integration by parts techniques are directly compared in the case of 1 -contact $n$-horizontal $(n+1)$ forms and we show that the two approaches are straightly equivalent, whilst in the case of 1-contact forms of lower degree the situation is more intricate, nevertheless, we obtain a comparable local splitting.

It is noteworthy that we obtain a compact and general expression for the splitting of a local variational morphism of any rank, which was not known in detail up to now (in [3] only a sketch of how the splitting would present in local coordinates is given).

Furthermore, an extension of the Krbek-Musilová splitting, as well as the construction of a local Interior Euler operator for contact forms of lower degree is obtained for any contact degree $k$. We work locally and the discussion of the globality of this splitting will be the subject of a separate paper.

Restricting to local splittings does not affect the main result obtained in the present paper, which is the definition, for the lower order case, of a suitable Residual operator (which even in the already known case of 1-contact $n$-horizontal $(n+1)$-forms is in general only locally defined). The aim is to construct, by a recurrence formula due to Olga Rossi, higher order (local) 
Lepage equivalents of a given Lagrangian.

Indeed, we recover the Krupka-Betounes Lepage equivalent for first order field theories and we obtain an elegant extension to the second order case. Again, globality properties of the latter deserve further study.

\section{Contact structure and geometric integra- tion by parts}

Prolongations of fibered manifolds are a basic tool for the geometric formulation of the calculus of variations. We recall the decomposition of pull-backs of differential forms on jet prolongations of fibered manifolds, performed by means of the jet projections and the holonomic lift of tangent vectors (a canonical construction which allows a splitting of the projection along the affine fibrations defining the contact structure of tangent vectors in two components with remarkable properties). Moreover the decomposition of forms leads to the introduction of the so-called contact forms, which reveal to be another fundamental concept in the calculus of variations. To fix notation we follow [13] ; other references on jet spaces are [20] and [8].

We recall that by a fibered manifold structure on a $C^{\infty}$ manifold $Y$ we mean a triplet $(Y, X, \pi)$, where $X$ is a $C^{\infty}$ manifold called the base and $\pi: Y \longrightarrow X$ is a surjective submersion of class $C^{\infty}$ called the projection. When dealing with local aspects of fibered manifolds, we will always use the so-called fibered charts (i.e. charts adapted to the fibration). Let $Y$ be a fibered manifold with base $X$ and projection $\pi$, let $n=\operatorname{dim} X$ and $m=\operatorname{dim} Y-n$. We denote by $J^{r} Y$, where $r \geq 0$ is any integer, the set of $r$-jets $J_{x}^{r} \gamma$ of $C^{r}$ sections of $Y$ with source $x \in X$ and target $y=\gamma(x) \in Y$ (for more details on jet spaces see [20] and [8]); we fix the notation $J^{0} Y=Y$. For any $s$ such that $0 \leq s \leq r$ we have surjective mappings, the canonical jet projections, $\pi_{s}^{r}: J^{r} Y \longrightarrow J^{s} Y$ and $\pi^{r}: J^{r} Y \longrightarrow X$, defined by $\pi_{s}^{r}\left(J_{x}^{r} \gamma\right)=$ $J_{x}^{s} \gamma, \pi^{r}\left(J_{x}^{r} \gamma\right)=x$. Let $(V, \psi), \psi=\left(x^{i}, y^{\sigma}\right)$, be a fibered chart on $Y$ and let $(U, \varphi), \varphi=\left(x^{i}\right)$, be the associated chart on $X$.

By setting $V^{r}=\left(\pi_{0}^{r}\right)^{-1}(V)$, a chart on the set $J^{r} Y$ associated with the fibered chart $(V, \psi)$ is given by $\left(V^{r}, \psi^{r}\right) \psi^{r}=\left(x^{i}, y^{\sigma}, y_{j_{1}}^{\sigma}, y_{j_{1} j_{2}}^{\sigma}, \ldots, y_{j_{1} j_{2} \ldots j_{r}}^{\sigma}\right)$, with $1 \leq i \leq n, \quad 1 \leq \sigma \leq m, 1 \leq j_{1} \leq j_{2} \leq \cdots \leq j_{k} \leq n, k=1,2,3, \ldots, r$. The set of associated charts $\left(V^{r}, \psi^{r}\right)$, such that the fibered charts $(V, \psi)$ constitute a smooth atlas on $Y$, is a smooth atlas on $J^{r} Y$. With this smooth 
structure $J^{r} Y$ is called the $r$-jet prolongation of the fibered manifold $Y$.

Let $Y$ be a fibered manifold with base $X$ and projection $\pi$. Let $\Xi$ be a $\pi$ projectable vector field on $Y$, expressed in a fibered chart $(V, \psi), \psi=\left(x^{i}, y^{\sigma}\right)$, by $\Xi=\xi^{i} \frac{\partial}{\partial x^{i}}+\Xi^{\sigma} \frac{\partial}{\partial y^{\sigma}}$, then its $s$-th prolongation $J^{s} \Xi$ is expressed in the associated chart $\left(V^{s}, \psi^{s}\right)$ by

$$
J^{s} \Xi=\xi^{i} \frac{\partial}{\partial x^{i}}+\Xi^{\sigma} \frac{\partial}{\partial y^{\sigma}}+\sum_{k=1}^{s} \sum_{j_{1} \leq j_{2} \leq \ldots \leq j_{k}} \Xi_{j_{1} j_{2} \ldots j_{k}}^{\sigma} \frac{\partial}{\partial y_{j_{1} j_{2} \ldots j_{k}}^{\sigma}},
$$

where $\Xi_{j_{1} j_{2} \ldots j_{k}}^{\sigma}=d_{j_{k}} \Xi_{j_{1} j_{2} \ldots j_{k-1}}^{\sigma}-y_{j_{1} j_{2} \ldots j_{k-1} i}^{\sigma} \frac{\partial \xi^{i}}{\partial x^{j_{k}}}$.

Let $J_{x}^{r+1} \gamma \in J^{r+1} Y$. To any tangent vector $\xi$ of $J^{r+1} Y$ at the point $J_{x}^{r+1} \gamma$ is assigned a tangent vector of $J^{r} Y$ at the point $\pi_{r}^{r+1}\left(J_{x}^{r+1} \gamma\right)=J_{x}^{r} \gamma$ by $h \xi:=$ $T J^{r} \gamma \circ T \pi^{r+1}(\xi)$. We get a vector bundle morphism $h: T J^{r+1} Y \longrightarrow T J^{r} Y$ over the jet projection $\pi_{r}^{r+1}$ called the horizontalization, and $h \xi$ is called the horizontal component of $\xi$. Let $\xi$ be given in a fibered chart $(V, \psi)$, $\psi=\left(x^{i}, y^{\sigma}\right)$ as $\xi=\left.\xi^{i} \frac{\partial}{\partial x^{i}}\right|_{J_{x}^{r+1} \gamma}+\left.\sum_{k=0}^{r+1} \sum_{j_{1} \leq j_{2} \leq \cdots \leq j_{k}} \Xi_{j_{1} j_{2} \ldots j_{k}}^{\sigma} \frac{\partial}{\partial y_{j_{1} j_{2} \ldots j_{k}}^{\sigma}}\right|_{J_{x}^{r+1} \gamma}$, then

$$
h \xi=\xi^{i} d_{i}:=\xi^{i}\left(\left.\frac{\partial}{\partial x^{i}}\right|_{J_{x}^{r} \gamma}+\left.\sum_{k=0}^{r} \sum_{j_{1} \leq j_{2} \leq \cdots \leq j_{k}} y_{j_{1} j_{2} \ldots j_{k} i}^{\sigma} \frac{\partial}{\partial y_{j_{1} j_{2} \ldots j_{k}}^{\sigma}}\right|_{J_{x}^{r} \gamma}\right),
$$

where the $i$-th formal derivative operator $d_{i}$ is a vector field along $\pi_{r}^{r+1}$.

We can assign to every tangent vector $\xi \in T_{J_{x}^{r+1} \gamma} J^{r+1} Y$ a tangent vector $p \xi \in T_{J_{x}^{r} \gamma} J^{r} Y$ by the decomposition $T \pi_{r}^{r+1}(\xi)=h \xi+p \xi$, where $p \xi$ is called the contact component of the vector $\xi$. Then

$$
p \xi=\left.\sum_{k=0}^{r} \sum_{j_{1} \leq j_{2} \leq \cdots \leq j_{k}}\left(\Xi_{j_{1 j_{2} \ldots j_{k}}}^{\sigma}-y_{j_{1} j_{2} \ldots j_{k} i}^{\sigma} \xi^{i}\right) \frac{\partial}{\partial y_{j_{1} j_{2} \ldots j_{k}}^{\sigma}}\right|_{J_{x}^{r} \gamma} .
$$

For any open set $W \subset Y, \Omega_{q}^{r} W$ denotes the $C^{\infty}$-module of $q$-forms on the open set $W^{r}=\left(\pi_{0}^{r}\right)^{-1}(W)$ in $J^{r} Y$, and $\Omega^{r} W$ is the exterior algebra of differential forms on $W^{r}$. In order to study the structure of the components of a form $\rho \in \Omega_{q}^{r} W$, it will be convenient to introduce a multi-index notation. A multi-index $I$ is an ordered $k$-tuple $I=\left(i_{1} i_{2} \ldots i_{k}\right)$, where $k=1,2, \ldots, r$ and the entries are indices such that $1 \leq i_{1}, i_{2}, \ldots, i_{k} \leq n$. The number $k$ is the lenght of $I$ and is denoted by $|I|$. If $1 \leq j \leq n$ is any integer, we denote by $I j$ the multi-index $I j=\left(i_{1} i_{2} \ldots i_{k} j\right)$. 
Geometric integration by parts and Lepage equivalents

The notion of horizontalization of vectors can be used to define a morphism $h: \Omega^{r} W \longrightarrow \Omega^{r+1} W$ of exterior algebras.

Let $\rho \in \Omega_{q}^{r} W$, with $q \geq 1$, and $J_{x}^{r+1} \gamma \in W^{r+1}$. Consider the pullback

$$
\begin{aligned}
& \left(\pi_{r}^{r+1}\right)^{*} \rho\left(J_{x}^{r+1} \gamma\right)\left(\xi_{1}, \xi_{2}, \ldots, \xi_{q}\right)= \\
& =\rho\left(J_{x}^{r} \gamma\right)\left(T \pi_{r}^{r+1}\left(\xi_{1}\right), T \pi_{r}^{r+1}\left(\xi_{2}\right), \ldots, T \pi_{r}^{r+1}\left(\xi_{q}\right)\right)
\end{aligned}
$$

on any tangent vectors $\xi_{1}, \xi_{2}, \ldots, \xi_{q}$ of $J^{r+1} Y$ at the point $J_{x}^{r+1} \gamma$. Decompose each of these vectors into the horizontal and contact components, $T \pi_{r}^{r+1}\left(\xi_{l}\right)=$ $h \xi_{l}+p \xi_{l}$, and set

$$
h \rho\left(J_{x}^{r+1} \gamma\right)\left(\xi_{1}, \xi_{2}, \ldots, \xi_{q}\right):=\rho\left(J_{x}^{r} \gamma\right)\left(h \xi_{1}, h \xi_{2}, \ldots, h \xi_{q}\right) .
$$

This formula defines a $q$-form $h \rho \in \Omega_{q}^{r+1} W$, while for 0 -forms $h f:=\left(\pi_{r}^{r+1}\right)^{*} f$. It follows that $h \rho\left(J_{x}^{r+1} \gamma\right)\left(\xi_{1}, \xi_{2}, \ldots, \xi_{q}\right)$ vanishes whenever at least one of the vectors is $\pi^{r+1}$-vertical. Thus, the $q$-form $h \rho$ must be $\pi^{r+1}$-horizontal. In particular $h \rho=0$ whenever $q \geq n+1$. The component $h \rho$ is called the horizontal component of $\rho$. We say that $\rho \in \Omega_{1}^{r} W$ is contact if $h \rho=0$. Let us now set, for $1 \leq k \leq q$,

$$
\begin{aligned}
& p_{k} \rho\left(J_{x}^{r+1} \gamma\right)\left(\xi_{1}, \xi_{2}, \ldots, \xi_{q}\right):= \\
& :=\frac{1}{k !(q-k) !} \sum_{\sigma \in \mathcal{P}_{q}}(-1)^{|\sigma|} \rho\left(J_{x}^{r} \gamma\right)\left(p \xi_{\sigma(1)}, \ldots, p \xi_{\sigma(k)}, h \xi_{\sigma(k+1)}, \ldots, h \xi_{\sigma(q)}\right)
\end{aligned}
$$

where $\mathcal{P}_{q}$ is the set of permutations of $q$ elements and $|\sigma|$ is the sign of the permutation $\sigma \in \mathcal{P}_{q}$. Note that for $k=0$, then we put $p_{0} \rho=h \rho$, while for 0 -forms $p_{k} f=0, k \geq 1$. In particular, given a $q$-form $\eta$

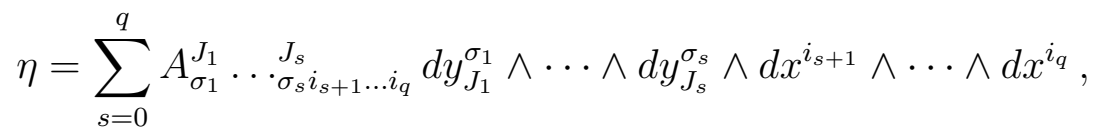

the $k$-contact component of $\eta$ has the chart expression

$$
p_{k} \eta=B_{\sigma_{1}}^{J_{1}} \cdots \sigma_{\sigma_{k} i_{k+1} \ldots i_{q}}^{J_{k}} \omega_{J_{1}}^{\sigma_{1}} \wedge \cdots \wedge \omega_{J_{k}}^{\sigma_{k}} \wedge d x^{i_{k+1}} \wedge \cdots \wedge d x^{i_{q}}
$$

with $\omega_{J}^{\sigma}=d y_{J}^{\sigma}-y_{J i}^{\sigma} d x^{i}$, and

$$
B_{\sigma_{1}}^{J_{1}} \ldots \sigma_{\sigma_{k} i_{k+1} \ldots i_{q}}^{J_{k}}=\sum_{s=k}^{q}\left(\begin{array}{l}
s \\
k
\end{array}\right) A_{\sigma_{1}}^{J_{1}} \ldots J_{\sigma_{k} \sigma_{k+1}}^{J_{k} J_{k+1}} \ldots \sigma_{\sigma_{s}\left[i_{s+1} \ldots i_{q}\right.}^{J_{s}} y_{J_{k+1} i_{k+1}}^{\sigma_{k+1}} \ldots y_{\left.J_{s} i_{s}\right]}^{\sigma_{s}}
$$


where the antisymmetrization in the right hand side of the last equation is performed only on the indices $i_{k+1} \ldots i_{s} i_{s+1} \ldots i_{q}$.

For any $\rho \in \Omega_{q}^{r} W, q \geq 0$, the canonical decomposition of the form $\rho$ is given as

$$
\left(\pi_{r}^{r+1}\right)^{*} \rho=h \rho+p_{1} \rho+p_{2} \rho+\cdots+p_{q} \rho .
$$

We can see that the canonical decomposition of forms gives rise to the splitting of the pull-back of the exterior derivative

$$
\left(\pi_{r}^{r+2}\right)^{*} d \rho=d_{H} \rho+d_{C} \rho:=\sum_{k=0}^{q} p_{k} d p_{k} \rho+\sum_{k=0}^{q} p_{k+1} d p_{k} \rho,
$$

and characterized by the identities $d_{H} \circ d_{H}=0, d_{C} \circ d_{C}=0, d_{C} \circ d_{H}=$ $-d_{H} \circ d_{C}$; furthermore, if $\rho$ is a $q$-form and $\eta$ is an $s$-form, both on $J^{r} Y$, then

$$
\begin{aligned}
& d_{H}(\rho \wedge \eta)=d_{H} \rho \wedge\left(\pi_{r}^{r+2}\right)^{*} \eta+(-1)^{q}\left(\pi_{r}^{r+2}\right)^{*} \rho \wedge d_{H} \eta \\
& d_{C}(\rho \wedge \eta)=d_{C} \rho \wedge\left(\pi_{r}^{r+2}\right)^{*} \eta+(-1)^{q}\left(\pi_{r}^{r+2}\right)^{*} \rho \wedge d_{C} \eta .
\end{aligned}
$$

\subsection{The interior Euler operator}

We recall some technical features of the interior Euler operator, seen as a tool which allows to pass in a univocal way from equivalence classes of local differential forms in the variational sequence, to (global) differential forms in the representation sequence. First we shortly recall the finite order variational sequence as introduced by Krupka in [12]. A complete description of this subject involves some topics of sheaf theory and sheaf cohomology; however, since our purpose is to make direct calculations on the representation of the variational sequence, we just refer to [13] for more details about those aspects. Then we shortly recall the notion of Lie derivative of forms with respect to a 'vector field along a map' and some results about integration by parts formulae which lead directly to the definition of the interior Euler operator. For more details and other related topics we refer to [9] and [16].

Let $\Omega_{q}^{r}, q \geq 0$, be the direct image of the sheaf of smooth $q$-forms over $J^{r} Y$ by the jet projection $\pi_{0}^{r}$. We denote by

$$
\Omega_{q, \mathrm{c}}^{r}=\left\{\begin{array}{l}
\operatorname{ker} p_{0} \quad \text { for } 1 \leq q \leq n, \\
\operatorname{ker} p_{q-n} \quad \text { for } n+1 \leq q \leq \operatorname{dim} J^{r} Y
\end{array}\right.
$$


the sheaf of contact $q$-forms, if $q \leq n$, or the sheaf of strongly contact $q$-forms, if $n+1 \leq q \leq \operatorname{dim} J^{r} Y$.

We set

$$
\Theta_{q}^{r}=\Omega_{q, \mathrm{c}}^{r}+d \Omega_{q-1, \mathrm{c}}^{r}
$$

where $d \Omega_{q-1, \mathrm{c}}^{r}$ is the image sheaf of $\Omega_{q-1, \mathrm{c}}^{r}$ by the exterior derivative $d$. The sequence of sheaves $\{0\} \rightarrow \Theta_{1}^{r} \rightarrow \cdots \rightarrow \Theta_{n}^{r} \rightarrow \Theta_{n+1}^{r} \rightarrow \cdots \rightarrow \Theta_{P}^{r} \rightarrow\{0\}$, with $d$ the exterior derivatives and $P$ being the maximal nontrivial degree, is an exact subsequence of the de Rham sequence. The acyclic resolution of the constant sheaf $\mathbb{R}_{Y}$ over $Y$, given by $\{0\} \rightarrow \mathbb{R}_{Y} \rightarrow \Omega_{*}^{r} / \Theta_{*}^{r}$, is called the variational sequence of order $r$. We denote the quotient mappings as $E_{q}^{r}:[\rho] \in \Omega_{q}^{r} / \Theta_{q}^{r} \longrightarrow E_{q}^{r}([\rho])=[d \rho] \in \Omega_{q+1}^{r} / \Theta_{q+1}^{r}$ Note that, in particular, the mappings $E_{n}^{r}$ and $E_{n+1}^{r}$ correspond to the Euler-Lagrange mapping and to the Helmholtz-Sonin mapping of calculus of variations, respectively.

Definition 2.1. Let $(V, \psi), \psi=\left(x^{i}, y^{\sigma}\right)$, be a fibered chart on $Y$ and let $\rho$ be a differential $q$-form on $J^{r} Y$. The Lie derivative of a $q$-form $\rho$ on with respect to a vector field $h \Xi$ along the map $\pi_{r}^{r+1}$ is given by

$$
\left.\left.£_{h \Xi}^{\pi_{r}^{r+1}} \rho=\left(\pi_{r}^{r+1}\right)_{h \Xi}^{*}(h \Xi\lrcorner d \rho\right)+d\left(\pi_{r}^{r+1}\right)_{h \Xi}^{*}(h \Xi\lrcorner \rho\right) .
$$

Here $\left(\pi_{r}^{r+1}\right)_{h \Xi}^{*}$ is a pull-back defined according to [9].

In particular, let $d_{i}$ be the $i$-th formal derivative operator seen as a (horizontal) vector field along a map. We have $£_{\mathrm{d}_{i}}^{\pi_{r}^{r+1}} d x^{j}=0, £_{\mathrm{d}_{i}}^{\pi_{r}^{r+1}} d y_{J}^{\sigma}=$ $d y_{J i}^{\sigma}, £_{\mathrm{d}_{i}}^{\pi_{r}^{r+1}} \omega_{J}^{\sigma}=\omega_{J i}^{\sigma}$, while for $f$ a zero form, we have $£_{\mathrm{d}_{i}}^{\pi_{r}^{r+1}} f=\frac{\partial f}{\partial x^{i}}+$ $\sum_{|J|=0}^{r} y_{J i}^{\sigma} \frac{\partial f}{\partial y_{J}^{\sigma}}=d_{i} f$.

Accordingly, by a slight abuse of notation, we will use at any degree the symbol $\mathrm{d}_{i}=£_{\mathrm{d}_{i}}^{\pi_{r}^{+1}}$, and we will call it the total derivative of forms with respect to the coordinate $x^{i}$.

We recall that the total derivatives of forms enjoy the following properties

1. the form $\mathrm{d}_{H} \rho$ can be locally decomposed as

$$
\mathrm{d}_{H} \rho=(-1)^{q} \mathrm{~d}_{i} \rho \wedge d x^{i}
$$

2. the Leibniz rule holds for total derivatives of the exterior product of forms $\rho$ and $\eta$

$$
\mathrm{d}_{i}(\rho \wedge \eta)=\mathrm{d}_{i} \rho \wedge \eta+\rho \wedge \mathrm{d}_{i} \eta
$$


3. let $(\bar{V}, \bar{\psi}), \bar{\psi}=\left(\bar{x}^{j}, \bar{y}^{\nu}\right)$, be a fibered chart on $Y$, such that $V \cap \bar{V} \neq \emptyset$ and let $\overline{\mathrm{d}}_{j}$ be the total derivative with respect to the coordinate $\bar{x}^{j}$. Then the transformation rule $\mathrm{d}_{i} \rho=\frac{\partial \bar{x}^{j}}{\partial x^{i}} \overline{\mathrm{d}}_{j} \rho$ holds.

4. the total derivatives commute, i.e.

$$
\mathrm{d}_{i} \mathrm{~d}_{j} \rho=\mathrm{d}_{j} \mathrm{~d}_{i} \rho
$$

This last property allows us to use the notation $\mathrm{d}_{J}=\mathrm{d}_{j_{s}} \circ \cdots \circ \mathrm{d}_{j_{1}}$, where $J=\left(j_{1} \ldots j_{s}\right)$ is a multi-index.

We consider the generalization of the integration by parts to differential forms based on the above concept of total derivative of forms and due to [9].

Let $(V, \psi), \psi=\left(x^{i}, y^{\sigma}\right)$, be a fibered chart on $Y$ and $\rho \in \Omega_{n+k}^{r} V$ a form. Let $\mathrm{p}_{k} \rho$ be expressed as

$$
\mathrm{p}_{k} \rho=\sum_{|J|=0}^{r} \omega_{J}^{\sigma} \wedge \eta_{\sigma}^{J}
$$

Then there exists the decomposition

$$
p_{k} \rho=\mathcal{I}(\rho)+p_{k} d p_{k} \mathcal{R}(\rho)
$$

where $\mathcal{I}$ is the interior Euler operator, $\mathcal{R}$ is the Residual operator, and $\mathcal{R}(\rho)$ is a local $k$-contact $(n+k-1)$-form.

There exists a unique decomposition as above such that $\mathcal{I}$ is $\mathbb{R}$-linear, which is therefore globally defined. In local coordinates we have

$$
\left.\mathcal{I}: \Omega_{n+k}^{r} W \ni \rho \longrightarrow \mathcal{I}(\rho)=\frac{1}{k} \omega^{\sigma} \wedge \sum_{|J|=0}^{r}(-1)^{|J|} \mathrm{d}_{J}\left(\frac{\partial}{\partial y_{J}^{\sigma}}\right\lrcorner p_{k} \rho\right) \in \Omega_{n+k}^{2 r+1} W
$$

Let $W \subset Y$ be an open set and let $\rho \in \Omega_{n+k}^{r} W, 1 \leq k \leq \operatorname{dim} J^{r} Y-n$, be a form. Then the following intrinsic properties uniquely characterize the interior Euler operator:

(a) $\left(\pi_{r}^{2 r+1}\right)^{*} \rho-\mathcal{I}(\rho) \in \Theta_{n+k}^{2 r+1} W ;$

(b) $\mathcal{I}\left(p_{k} d p_{k} \mathcal{R}(\rho)\right)=0$;

(c) $\mathcal{I}^{2}(\rho)=\left(\pi_{2 r+1}^{4 r+3}\right)^{*} \mathcal{I}(\rho)$;

(d) $\operatorname{ker}(\mathcal{I})=\Theta_{n+k}^{r} W$. 


\subsection{Variational morphisms and canonical splittings}

We now recall shortly the definition and the basic properties of variational morphisms; see [3]. In view of a comparison with the Krbek-Musilová geometric integration by parts, we discuss their algorithmic splitting properties, which correspond to the possibility of performing a global and covariant integration by parts. We distinguish the case of codegree $s=0$ from the case $0<s \leq n$ and include some results about the uniqueness properties of the aforementioned splittings.

Definition 2.2. Let $\mathcal{E}=\left(E, X, \tilde{\pi}, \mathbb{R}^{l}\right)$ be a vector bundle and $\pi: Y \longrightarrow X$ an arbitrary fiber bundle, both over $X$, with $\operatorname{dim} X=n$, and let $A_{q}(X)$ denote the bundle of q-forms on $X$. Let $t, r$ and $s$ be integers. A bundle morphism

$$
\mathbb{V}: J^{t} Y \longrightarrow\left(J^{r} \mathcal{E}\right)^{*} \otimes A_{n-s}(X)
$$

is called a variational $\mathcal{E}$-morphism on $Y$. The (minimal) integer $t$ is called the order of $\mathbb{V}, r$ is called the rank and $(n-s)$ is called the degree of $\mathbb{V}$ (being $s$ the codegree).

A fibered connection on $\mathcal{E}$ (i.e. a linear connection $\Gamma_{b i}^{a}$ on $X$ and a connection $\Gamma_{B i}^{A}$ on $\left.\mathcal{E}\right)$ induces on $\left(J^{r} \mathcal{E}\right)^{*} \otimes A_{n-s}(X)$ a set of local fibered coordinates $\left(x^{i} ; \hat{v}_{A}^{i_{1} \ldots i_{s}}, \ldots, \hat{v}_{A}^{i_{1} \ldots i_{s} j_{1} \ldots j_{r}}\right)$ so that a variational morphism $\mathbb{V}$ can be locally given there as

$$
<\mathbb{V} \mid J^{r} \Xi>=\frac{1}{s !}\left[\hat{v}_{A}^{i_{1} \ldots i_{s}} \hat{\Xi}^{A}+\hat{v}_{A}^{i_{1} \ldots i_{s} j} \hat{\Xi}_{j}^{A}+\cdots+\hat{v}_{A}^{i_{1} \ldots i_{s} j_{1} \ldots j_{r}} \hat{\Xi}_{j_{1} \ldots j_{r}}^{A}\right] \otimes d s_{i_{1} \ldots i_{s}}
$$

where $\left.\left.\left.d s_{i_{1} \ldots i_{s}}=\frac{\partial}{\partial x^{i_{s}}}\right\lrcorner \ldots\right\lrcorner \frac{\partial}{\partial x^{i_{1}}}\right\lrcorner d s$, if $d s$ is the volume density on the base manifold $X$. Each coefficient $\mathbb{V}_{m}=\frac{1}{s !} \hat{v}_{A}^{i_{1} \ldots i_{s} j_{1} \ldots j_{m}}$ of order $0 \leq m \leq r$ is the coefficient of a global variational morphism, called the m-rank term of $\mathbb{V}$ (if $m=r$ it is called the highest rank term of $\mathbb{V})$.

Let now $\mathbb{Q}: J^{t} Y \longrightarrow A_{n-s}(X)$ be a morphism of rank $r=0$. The divergence of $\mathbb{Q}$ is the variational morphism $\operatorname{Div}(\mathbb{Q}): J^{t+1} Y \longrightarrow A_{n-s+1}(M)$ such that

$$
\operatorname{Div}(\mathbb{Q}) \circ J^{t+1} \sigma=d\left(\mathbb{Q} \circ J^{t} \sigma\right)
$$

for each section $\sigma: X \longrightarrow Y$.

Variational morphisms admit canonical and algorithmic splittings corresponding to global and covariant integration by parts. We distinguish two cases. 
Geometric integration by parts and Lepage equivalents

- the case of codegree $s=0$.

Let $\mathbb{V}: J^{t} Y \longrightarrow\left(J^{r} \mathcal{E}\right)^{*} \otimes A_{n}(X)$ be a variational $\mathcal{E}$-morphism of codegree $s=0$. Then we can define two global variational $\mathcal{E}$-morphisms

$$
\begin{aligned}
& \mathbb{E} \equiv \mathbb{E}(\mathbb{V}): J^{t+r} C \longrightarrow \mathcal{E}^{*} \otimes A_{n}(X) \\
& \mathbb{T} \equiv \mathbb{T}(\mathbb{V}): J^{t+r-1} C \longrightarrow\left(J^{r-1} \mathcal{E}\right)^{*} \otimes A_{n-1}(X)
\end{aligned}
$$

such that the following splitting property holds true:

$$
<\mathbb{V}\left|J^{r} \Xi>=<\mathbb{E}\right| \Xi>+\operatorname{Div}\left(<\mathbb{T} \mid J^{r-1} \Xi>\right)
$$

for each section $\Xi$ of $\mathcal{E}$. The variational morphism $\mathbb{E}$ and $\mathbb{T}$ are called the volume part and the boundary part of $\mathbb{V}$, respectively.

In particular, we locally have:

$$
<\mathbb{E} \mid \Xi>=\left[\left(\hat{v}_{A}-\nabla_{j_{1}} \hat{v}_{A}^{j_{1}}+\cdots+(-1)^{r} \nabla_{j_{1} \ldots j_{r}} \hat{v}_{A}^{j_{1} \ldots j_{r}}\right) \Xi^{A}\right] \otimes d s
$$

and

$$
<\mathbb{T} \mid J^{r-1} \Xi>=\left[\hat{t} \hat{\Xi}_{A} \hat{\Xi}^{A}+\hat{t}_{A}^{i j_{1}} \hat{\Xi}_{j_{1}}^{A}+\cdots+\hat{t}_{A}^{j_{1} \ldots j_{r-1}} \hat{\Xi}_{j_{1} \ldots j_{r-1}}^{A}\right] \otimes d s_{i}
$$

where the coefficients of $\mathbb{T}$ are given by the recurrence relations

$$
\begin{aligned}
& \hat{t}_{A}^{i j_{1} \ldots j_{r-1}}=\hat{v}_{A}^{i j_{1} \ldots j_{r-1}} \\
& \hat{t}_{A}^{i j_{1} \ldots j_{r-2}}=\hat{v}_{A}^{i j_{1} \ldots j_{r-2}}-\nabla_{l} \hat{t}_{A}^{l i j_{1} \ldots j_{r-2}} \\
& \cdots \\
& \hat{t}_{A}^{i}=\hat{v}_{A}^{i}-\nabla_{l} \hat{t}_{A}^{l i} .
\end{aligned}
$$

A similar splitting formula can be obtained for variational morphisms of higher codegree. The expressions in coordinates are in this case more complicated due to the presence of some antisymmetries in the indices of the local coefficients; see in particular [3], p. 161-162. The concept of a reduced morphism (with respect to a fibered connection) is shown to be necessary.

Definition 2.3. Let $\mathbb{V}: J^{t} Y \longrightarrow\left(J^{r} \mathcal{E}\right)^{*} \otimes A_{n-s}(X)$ be a variational morphism.

Let $\mathbb{V}_{m}=\frac{1}{s !}\left(\hat{v}_{A}^{i_{1} \ldots i_{s} j_{1} \ldots j_{m}}\right) \otimes d s_{i_{1} \ldots i_{s}}$ be the coefficient of its term of rank $0 \leq m \leq r$.

The term $\mathbb{V}_{m}$ is said to be reduced with respect to the fibered connection $\left(\Gamma_{b i}^{a}, \Gamma_{B i}^{A}\right)$ if $\hat{v}_{A}^{\left[i_{1} \ldots i_{s} j_{1}\right] j_{2} \ldots j_{m}}=0$. The variational morphism $\mathbb{V}$ is reduced if all its terms are reduced. 
Notice that when $n=\operatorname{dim}(X)=1$, e.g. in the case of Mechanics, all variational morphisms are reduced. However, the property of being reduced in general depends on the choice of the a fibered connection, whenever the rank is at least two.

- the case of codegree $s \geq 1$.

Let now $\mathbb{V}: J^{t} Y \longrightarrow\left(J^{r} \mathcal{E}\right)^{*} \otimes A_{n-s}(X)$ be a global variational $\mathcal{E}$ morphism of codegree $s \geq 1$. Then we can define two global variational $\mathcal{E}$-morphisms

$$
\begin{aligned}
& \mathbb{E} \equiv \mathbb{E}(\mathbb{V}): J^{t+r} C \longrightarrow\left(J^{r} \mathcal{E}\right)^{*} \otimes A_{n-s}(X) \\
& \mathbb{T} \equiv \mathbb{T}(\mathbb{V}): J^{t+r-1} C \longrightarrow\left(J^{r-1} \mathcal{E}\right)^{*} \otimes A_{n-s-1}(X)
\end{aligned}
$$

where $\mathbb{E}$ is a reduced variational morphism and such that the following holds true:

$$
<\mathbb{V}\left|J^{r} \Xi>=<\mathbb{E}\right| J^{r} \Xi>+\operatorname{Div}\left(<\mathbb{T} \mid J^{r-1} \Xi>\right)
$$

for each section $\Xi$ of $\mathcal{E}$. Only a sketch of the expression in local coordinates of the volume part $\mathbb{E}$ and the boundary part $\mathbb{T}$ can be found in [3].

Let a fibered connection be fixed; the volume part is uniquely determined, while the boundary part is determined modulo a divergenceless term. When $r \geq 2$ one can proceed by further splitting

$$
<\mathbb{T}\left|J^{r-1} \Xi>=<\mathbb{S}\right| J^{r-1} \Xi>+\operatorname{Div}\left(<\mathbb{Q} \mid J^{r-2} \Xi>\right),
$$

where the variational morphism $\mathbb{S}: J^{t+2 r-2} Y \longrightarrow\left(J^{r-1} \mathcal{E}\right)^{*} \otimes A_{n-s-1}(X)$ is reduced by construction and uniquely determined.

\section{Comparison of the two approaches and new results}

We present some original results which clarify the similarities and differences between the two integration by parts methods described above.

The basic idea is that 1-contact forms of degree $n+1$ can be seen as variational morphisms and, viceversa, to each variational morphism a 1-contact 
form of degree $n+1$ can be associated. In Proposition 3.1, we prove the equivalence of decompositions (2) and (41) for 1-contact $(n+1)$-forms (which we shall call top forms, because they are of the highest horizontal degree), seen as variational morphisms of codegree $s=0$.

\subsection{Contact forms as (local) variational morphisms}

Consider an arbitrary bundle $\pi: Y \longrightarrow X$ with $n=\operatorname{dim} X$. Let $U \subseteq X$ be an open subset and let $W=\pi^{-1}(U)$ be the "tube" over $U$. Consider $\rho \in \Omega_{q}^{r} W$ a 1-contact $q$-form on $W^{r}=\left(\pi_{0}^{r}\right)^{-1}(W)$, with $q \leq n+1$. Then, if $\left(W^{r}, \psi^{r}\right)$ is a local chart on $J^{r} Y$ associated with the fibered chart $(W, \psi), \psi=\left(x^{i}, y^{\sigma}\right)$ on $Y$, we can write

$$
p_{1} \rho=\sum_{|J|=0}^{r} \omega_{J}^{\sigma} \wedge \eta_{\sigma}^{J} \in \Omega_{q}^{r+1} W
$$

where $\eta_{\sigma}^{J}$ are horizontal $(q-1)$-forms defined on $W^{r+1}$ and thence can be expressed as

$$
\eta_{\sigma}^{J}=A_{\sigma}^{i_{1} \ldots i_{s} J}\left(J^{r+1} y\right) d s_{i_{1} \ldots i_{s}}, \quad s=n-(q-1) .
$$

Now, considering the vector bundle $V(W)$ whose sections are vertical vector fields over $W \rightarrow U$ and recalling that $J^{r} V(W) \cong V\left(J^{r} W\right)$, we can define according to Definition 2.2 a variational morphism $\mathbb{V}_{\rho}: J^{r+1} W \longrightarrow$ $\left(J^{r} V(W)\right)^{*} \otimes A_{s}(U)$ such that:

$$
\begin{aligned}
& \left.<\mathbb{V}_{\rho} \mid J^{r} \Xi>=J^{r} \Xi\right\lrcorner p_{1} \rho=\left[\sum_{|J|=0}^{r} A_{\sigma}^{i_{1} \ldots i_{s} J} \Xi_{J}^{\sigma}\right] \otimes d s_{i_{1} \ldots i_{s}}= \\
& =\left[A_{\sigma}^{i_{1} \ldots i_{s}} \Xi^{\sigma}+A_{\sigma}^{i_{1} \ldots i_{s} j_{1}} \Xi_{j_{1}}^{\sigma}+\cdots+A_{\sigma}^{i_{1} \ldots i_{s} j_{1} \ldots j_{r}} \Xi_{j_{1} \ldots j_{r}}^{\sigma}\right] \otimes d s_{i_{1} \ldots i_{s}}
\end{aligned}
$$

for every vertical vector field $\Xi: W \longrightarrow V(W)$.

The advantage of this approach consists in the possibility of working on contact forms (although only in the particular case of 1-contact forms of degree at most $n+1$ ) using the tools of the theory of variational morphisms and returning back to forms at the end of the manipulation.

It appears that the above identification of 1-contact forms with variational morphisms holds true up to $(n+1)$-forms and, indeed, this could be related to the non-uniqueness of the source forms providing the so-called Helmholtz conditions. In fact, as discussed in [16] (pag. 32), this feature appears for $k$-contact $n$-horizontal $(n+k)$-forms with $k \geq 2$. 
Geometric integration by parts and Lepage equivalents

\subsection{Comparison for top forms}

In this section we directly compare the two integration by parts procedures.

As a first step, in the following proposition we prove that the splitting (4) of $p_{1} \rho$, seen as a variational morphism $\mathbb{V}_{\rho}$ of codegree $s=0$,

$$
<\mathbb{V}_{\rho}\left|J^{r} \Xi>=<\mathbb{E}\right| \Xi>+\operatorname{Div}\left(<\mathbb{T} \mid J^{r-1} \Xi>\right)
$$

and the decomposition

$$
p_{1} \rho=\mathcal{I}(\rho)+d_{H} \mathcal{R}(\rho)
$$

give the same terms.

Proposition 3.1. Given $\rho \in \Omega_{n+1}^{r} W$ a 1-contact $(n+1)$-form. For every section $\Xi: W \longrightarrow V(W)$,

$$
\left.<\mathbb{V}_{\rho} \mid J^{r} \Xi>=J^{r} \Xi\right\lrcorner p_{1} \rho
$$

and

$$
\left.\left.<\mathbb{E} \mid \Xi>=J^{r} \Xi\right\lrcorner \mathcal{I}(\rho), \quad \operatorname{Div}\left(<\mathbb{T} \mid J^{r-1} \Xi>\right)=J^{r} \Xi\right\lrcorner d_{H} \mathcal{R}(\rho) .
$$

Proof. Step 1. The fact that $\left.\langle\mathbb{E} \mid \Xi\rangle=J^{r} \Xi\right\lrcorner \mathcal{I}(\rho)$ follows directly from the definition of each side of the equation. In fact, since $W$ is a single coordinate domain, we can always choose the fibered connection whose coefficients are all null, then the covariant derivatives reduce to total derivatives and the variational morphism $\mathbb{E}$ takes the form:

$$
<\mathbb{E} \mid \Xi>=\left[\left(A_{\sigma}-d_{j_{1}} A_{\sigma}^{j_{1}}+\cdots+(-1)^{r} d_{j_{1} \ldots j_{r}} A_{\sigma}^{j_{1} \ldots j_{r}}\right) \Xi^{\sigma}\right] \otimes d s .
$$

On the other hand, from the definition of the interior Euler operator, we have

$$
\begin{aligned}
\mathcal{I}(\rho)=\omega^{\sigma} & \wedge \sum_{|J|=0}^{r}(-1)^{|J|} \mathrm{d}_{J} \eta_{\sigma}^{J}=\omega^{\sigma} \wedge\left(\sum_{|J|=0}^{r}(-1)^{|J|} d_{J} A_{\sigma}^{J}\right) d s= \\
& =\omega^{\sigma} \wedge\left(A_{\sigma}-d_{j_{1}} A_{\sigma}^{j_{1}}+\cdots+(-1)^{r} d_{j_{1} \ldots j_{r}} A_{\sigma}^{j_{1} \ldots j_{r}}\right) d s .
\end{aligned}
$$

Step 2. In order to compare $\left.J^{r} \Xi\right\lrcorner d_{H} \mathcal{R}(\rho)$ with $\operatorname{Div}\left(<\mathbb{T} \mid J^{r-1} \Xi>\right)$ we need to compute explicitly the Residual operator $\mathcal{R}(\rho)$. We now write $p_{1} \rho=$ $\sum_{|I|=0}^{r} \mathrm{~d}_{I}\left(\omega^{\sigma} \wedge \xi_{\sigma}^{I}\right)$, where

$$
\xi_{\sigma}^{I}=\sum_{|J|=0}^{r-|I|}(-1)^{|J|}\left(\begin{array}{c}
|J|+|I| \\
|J|
\end{array}\right) \mathrm{d}_{J} \eta_{\sigma}^{I J}=\left(\sum_{|J|=0}^{r-|I|}(-1)^{|J|}\left(\begin{array}{c}
|J|+|I| \\
|J|
\end{array}\right) \mathrm{d}_{J} A_{\sigma}^{I J}\right) d s .
$$


The summand $\omega^{\sigma} \wedge \xi_{\sigma}$ is the interior Euler operator, so we consider only the remaining terms $\sum_{|I|=1}^{r} \mathrm{~d}_{I}\left(\omega^{\sigma} \wedge \xi_{\sigma}^{I}\right)$. Each form $\omega^{\sigma} \wedge \xi_{\sigma}^{I}$ is a 1-contact $(n+1)$-form and thence can be recast as $\omega^{\sigma} \wedge \xi_{\sigma}^{I}=\chi^{I} \wedge d s$, where $\chi^{I}$ is a 1 -contact 1-form locally given as

$$
\chi^{I}=\left(\sum_{|J|=0}^{r-|I|}(-1)^{|J|}\left(\begin{array}{c}
|J|+|I| \\
|J|
\end{array}\right) \mathrm{d}_{J} A_{\sigma}^{I J}\right) \omega^{\sigma} .
$$

Finally, the Residual operator is defined by

$$
\begin{aligned}
& \mathcal{R}(\rho)=\sum_{|I|=0}^{r-1}(-1)^{1} \mathrm{~d}_{I} \chi^{i I} \wedge d s_{i}= \\
& =\sum_{|I|=0}^{r-1}-\mathrm{d}_{I}\left(\sum_{|J|=0}^{r-|i I|}(-1)^{|J|}\left(\begin{array}{c}
|J|+|i I| \\
|J|
\end{array}\right) \omega^{\sigma} \mathrm{d}_{J} A_{\sigma}^{i I J}\right) \wedge d s_{i} .
\end{aligned}
$$

Now, from the second equation above, we compute the coefficients of the forms $\omega_{L}^{\sigma} \wedge d s_{i}$ according to the length of the multi-index $L$ :

$|L|=r-1$. The only contribution to this coefficient comes from setting $|I|=$ $r-1$ and applying all the total derivatives of forms $\mathrm{d}_{I}$ to $\omega^{\sigma}$, thus obtaining as coefficient $A_{\sigma}^{i L}$.

$|L|=r-2$. One contribution comes from setting $|I|=r-2$ and applying all the total derivatives of forms $\mathrm{d}_{I}$ to $\omega^{\sigma}$, getting the coefficient

$$
-\left(A_{\sigma}^{i L}-r d_{l} A_{\sigma}^{l i L}\right)
$$

another contribution comes from setting $|I|=r-1$ and applying $r-2$ total derivatives to $\omega^{\sigma}$ and one to $A_{\sigma}^{i I}$, thus obtaining

$$
-\left(\begin{array}{c}
r-1 \\
1
\end{array}\right) d_{l} A_{\sigma}^{l i L}
$$

summing together these two terms, we get the coefficient

$$
-\left(A_{\sigma}^{i L}-d_{l} A_{\sigma}^{l i L}\right) .
$$


Geometric integration by parts and Lepage equivalents

$|L|=r-3$. We can take $|I|=r-3$ and apply all the total derivatives to $\omega^{\sigma}$, getting a term

$$
-A_{\sigma}^{i L}+\left(\begin{array}{c}
r-1 \\
1
\end{array}\right) d_{l} A_{\sigma}^{l i L}-\left(\begin{array}{l}
r \\
2
\end{array}\right) d_{l k} A_{\sigma}^{l k i L}
$$

another contribution comes from setting $|I|=r-2$ and applying only $r-3$ derivatives to $\omega^{\sigma}$, getting a term

$$
-\left(\begin{array}{c}
r-2 \\
1
\end{array}\right) d_{l} A_{\sigma}^{l i L}+\left(\begin{array}{c}
r \\
1
\end{array}\right)\left(\begin{array}{c}
r-2 \\
1
\end{array}\right) d_{l k} A_{\sigma}^{l k i L},
$$

finally, we can take $|I|=r-1$ and apply $r-3$ derivatives to $\omega^{\sigma}$, getting a term

$$
-\left(\begin{array}{c}
r-1 \\
2
\end{array}\right) d_{l k} A_{\sigma}^{l k i L}
$$

summing all together these contributions we obtain the following coefficient

$$
-\left(A_{\sigma}^{i L}-d_{l} A_{\sigma}^{l i L}+d_{l k} A_{\sigma}^{l k i L}\right) .
$$

In general, we thus get

$$
\begin{aligned}
|L|=r- & m, 1 \leq m \leq r . \\
& -\left(A_{\sigma}^{i L}-d_{j_{1}} A_{\sigma}^{i L j_{1}}+\ldots(-1)^{m-1} d_{j_{1} j_{2} j_{3} \ldots j_{m-1}} A_{\sigma}^{i L j_{1} j_{2} j_{3} \ldots j_{m-1}}\right) .
\end{aligned}
$$

It appears clear then that the coefficients of the forms $\omega_{L}^{\sigma} \wedge d s_{i}$ are defined by the same recurrence relations (5) which express the components $t_{\sigma}^{i J}$ of the variational morphism $\mathbb{T}$, except for the sign. Thence we can write for the Krbek-Musilová Residual operator

$$
\mathcal{R}(\rho)=\sum_{|J|=0}^{r-1}-t_{\sigma}^{i J} \omega_{J}^{\sigma} \wedge d s_{i} .
$$

Therefore, $d_{H} \mathcal{R}(\rho)=p_{1} d p_{1} \mathcal{R}(\rho)=\sum_{|J|=0}^{r-1}\left(d_{i} t_{\sigma}^{i J} \omega_{J}^{\sigma} \wedge d s+t_{\sigma}^{i J} \omega_{J i}^{\sigma} \wedge d s\right)$, and 
since $\Xi_{J i}^{\sigma}=d_{i} \Xi_{J}^{\sigma}$, we finally get

$$
\begin{aligned}
& \left.J^{r} \Xi\right\lrcorner d_{H} \mathcal{R}(\rho)=\sum_{|J|=0}^{r-1}\left(d_{i} t_{\sigma}^{i J} \Xi_{J}^{\sigma}+t_{\sigma}^{i J} \Xi_{J i}^{\sigma}\right) \wedge d s= \\
& =\sum_{|J|=0}^{r-1}\left(d_{i} t_{\sigma}^{i J} \Xi_{J}^{\sigma}+t_{\sigma}^{i J} d_{i} \Xi_{J}^{\sigma}\right) \wedge d s \\
& =d_{i}\left(\sum_{|J|=0}^{r-1} t_{\sigma}^{i J} \Xi_{J}^{\sigma}\right) \wedge d s=\operatorname{Div}\left(<\mathbb{T} \mid J^{r-1} \Xi>\right) .
\end{aligned}
$$

q.e.d.

Remark 3.1. Note that, since $d_{H} d s_{i_{1} \ldots i_{s}}=0$, the latter equation can be generalized to hold true for every $0 \leq s \leq n$. Indeed, let $\mathbb{T}: J^{t} Y \longrightarrow$ $\left(J^{r-1} V(Y)\right)^{*} \otimes A_{n-s}(X)$ be a variational morphism according to Definition 2.2, with

$$
<\mathbb{T} \mid J^{r-1} \Xi>=\left(\sum_{|J|=0}^{r-1} t_{\sigma}^{i_{1} \ldots i_{s} J} \Xi_{J}^{\sigma}\right) \wedge d s_{i_{1} \ldots i_{s}}
$$

for any vertical vector field $\Xi$ over $Y$. Then there is a correspondence between $\mathbb{T}$ and a $(n-s)$-horizontal 1-contact $(n-s+1)$-form $\tilde{\mathcal{R}}$ such that

$$
\left.\operatorname{Div}\left(<\mathbb{T} \mid J^{r-1} \Xi>\right)=J^{r} \Xi\right\lrcorner d_{H} \tilde{\mathcal{R}}
$$

where $\tilde{\mathcal{R}}$ is defined by

$$
\tilde{\mathcal{R}}=\left(\sum_{|J|=0}^{r-1}-t_{\sigma}^{i_{1} \ldots i_{s} J} \omega_{J}^{\sigma}\right) \wedge d s_{i_{1} \ldots i_{s}} .
$$

In spite of the suggestive notation, $\tilde{\mathcal{R}}$ does not deal with a Residual operator at this stage.

\section{The main result: comparison for lower de- gree forms}

The more intricate case of 1-contact $(n-s+1)$-forms, seen as variational morphisms of codegree $0<s \leq n$, is discussed. 
First we show that, in general, for $k$-contact $(n-s)$-horizontal $(n-s+k)$ forms, with an adequate manipulation, it is possible to obtain a decomposition analogous to (2). In Proposition 4.1 we characterize the boundary term by a local differential operator which extends to the case of $k$-contact forms of lower degree the Krbek-Musilova's Residual operator. It is defined for forms of lower degree of any order of contactness $k$.

In Proposition 4.2 we show that, when we restrict to $k=1$, this decomposition is indeed equivalent to the application of a "canonical splitting"like algorithm to the corresponding variational morphism. We also define a suitable extension of the interior Euler operator to 1-contact forms of lower degree. In this specific case, we also provide some examples, in particular for $r=1,0 \leq s<n$ and for $r=2, s=1$, where we compare this decomposition with (6); the latter example shows that the difference between the respective boundary terms is indeed a local divergence, dealing with the splitting (77).

Let $\pi: Y \longrightarrow X$, and $U \subseteq X$ an open subset and $W=\pi^{-1}(U)$. Let $\rho \in \Omega_{n-s+k}^{r} W$ be a $(n-s)$-horizontal $k$-contact $(n-s+k)$-form defined on the $r$-order jet prolongation $W^{r}$ of $W$. In a local fibered chart $\psi^{r}=\left(x^{i}, y^{\sigma}, y_{I}^{\sigma}\right)$, we can write:

$$
p_{k} \rho=\sum_{|J|=0}^{r} \omega_{J}^{\sigma} \wedge \eta_{\sigma}^{J} \in \Omega_{n-s+k}^{r+1} W
$$

where $\eta_{\sigma}^{J}$ are local $(n-s)$-horizontal $(k-1)$-contact $(n-s+k-1)$-forms defined on $W^{r+1}$. Again, according to [9], we can rewrite

$$
p_{k} \rho=\sum_{|J|=0}^{r} \omega_{J}^{\sigma} \wedge \eta_{\sigma}^{J}=\sum_{|I|=0}^{r} \mathrm{~d}_{I}\left(\omega^{\sigma} \wedge \xi_{\sigma}^{I}\right)=\omega^{\sigma} \wedge \xi_{\sigma}+\sum_{|I|=1}^{r} \mathrm{~d}_{I}\left(\omega^{\sigma} \wedge \xi_{\sigma}^{I}\right)
$$

where

$$
\xi_{\sigma}^{I}=\sum_{|J|=0}^{r-|I|}(-1)^{|J|}\left(\begin{array}{c}
|J|+|I| \\
|J|
\end{array}\right) d_{J} \eta_{\sigma}^{I J} .
$$

In analogy with Step 2 of the above Proposition (which can be recovered for $k=1, s=0)$ we work out the term $\sum_{|I|=1}^{r} \mathrm{~d}_{I}\left(\omega^{\sigma} \wedge \xi_{\sigma}^{I}\right)$. Each form $\omega^{\sigma} \wedge \xi_{\sigma}^{I}$ is a $(n-s)$-horizontal $k$-contact $(n-s+k)$-form, thus it can be recast in the following manner:

$$
\omega^{\sigma} \wedge \xi_{\sigma}^{I}=\chi^{i_{1} \ldots i_{s} I} \wedge d s_{i_{1} \ldots i_{s}}
$$


where the $\chi^{i_{1} \ldots i_{s} I}$ are local $k$-contact $k$-forms. Renaming the multi-index $I$ and extracting an antisymmetric part, we obtain:

$$
\begin{aligned}
& \sum_{|I|=1}^{r} \mathrm{~d}_{I}\left(\omega^{\sigma} \wedge \xi_{\sigma}^{I}\right)=\sum_{|I|=0}^{r-1} \mathrm{~d}_{i} \mathrm{~d}_{I} \chi^{i_{1} \ldots i_{s} i I} \wedge d s_{i_{1} \ldots i_{s}}= \\
& =\sum_{|I|=0}^{r-1} \mathrm{~d}_{i} \mathrm{~d}_{I}\left(\chi^{i_{1} \ldots i_{s} i I}-\chi^{\left[i_{1} \ldots i_{s} i\right] I}\right) \wedge d s_{i_{1} \ldots i_{s}}+\mathrm{d}_{i} \sum_{|I|=0}^{r-1} \mathrm{~d}_{I} \chi^{\left[i_{1} \ldots i_{s} i\right] I} \wedge d s_{i_{1} \ldots i_{s}} .
\end{aligned}
$$

Lemma 4.1. We have

$$
\sum_{|I|=0}^{r-1} d_{i} d_{I}\left(\chi^{i_{1} \ldots i_{s} i I}-\chi^{\left[i_{1} \ldots i_{s} i\right] I}\right) \wedge d s_{i_{1} \ldots i_{s}} \equiv 0 .
$$

Proof. Let us consider the terms

$$
\mathrm{d}_{i} \chi^{i_{1} \ldots i_{s} i I} \wedge d s_{i_{1} \ldots i_{s}}
$$

here a sum on the index $i$ is understood, let us write it explicitly in order to manipulate it:

$$
\mathrm{d}_{1} \chi^{i_{1} \ldots i_{s} 1 I} \wedge d s_{i_{1} \ldots i_{s}}+\mathrm{d}_{2} \chi^{i_{1} \ldots i_{s} 2 I} \wedge d s_{i_{1} \ldots i_{s}}+\mathrm{d}_{3} \chi^{i_{1} \ldots i_{s} 3 I} \wedge d s_{i_{1} \ldots i_{s}}+\ldots
$$

Let $i_{p} \neq i, 1 \leq p \leq s$, we have

$$
d s_{i_{1} \ldots i_{s}}=d x^{i} \wedge d s_{i_{1} \ldots i_{s} i}
$$

which holds for each $i \neq i_{1}, \ldots, i_{s}$ and is intended without summation over $i$. Let us substitute for each convenient index in the summation. We get

$$
\mathrm{d}_{1} \chi^{i_{1} \ldots i_{s} 1 I} \wedge d x^{1} \wedge d s_{i_{1} \ldots i_{s} 1}+\cdots+\mathrm{d}_{i} \chi^{i_{1} \ldots i_{s} i I} \wedge d x^{i} \wedge d s_{i_{1} \ldots i_{s} i}+\ldots
$$

Now, by rearranging the summation (see in detail the procedure in the proof of next Proposition) note that in $d s_{i_{1} \ldots i_{s} i}$ the indices $i_{1} \ldots i_{s} i$ are antisymmetric for each $i$ therefore necessarily each of the coefficients must satisfy $\chi^{i_{1} \ldots i_{s} 1 I}=\chi^{\left[i_{1} \ldots i_{s} 1\right] I}, \chi^{i_{1} \ldots i_{s} 2 I}=\chi^{\left[i_{1} \ldots i_{s} 2\right] I}, \ldots$, therefore for each $i \neq i_{1}, \ldots, i_{s}$ $\chi^{i_{1} \ldots i_{s} i I}=\chi^{\left[i_{1} \ldots i_{s} i\right] I}$. Thus if $i_{p} \neq i, 1 \leq p \leq s$,

$$
\begin{array}{r}
\sum_{|I|=0}^{r-1} \mathrm{~d}_{i} \mathrm{~d}_{I}\left(\chi^{i_{1} \ldots i_{s} i I}-\chi^{\left[i_{1} \ldots i_{s} i\right] I}\right) \wedge d s_{i_{1} \ldots i_{s}}= \\
\sum_{|I|=0}^{r-1} \mathrm{~d}_{j} \mathrm{~d}_{I}\left(\chi^{\left[i_{1} \ldots i_{s} i\right] I}-\chi^{\left[i_{1} \ldots i_{s} i\right] I}\right) \wedge d x^{j} \wedge d s_{i_{1} \ldots i_{s} i} \equiv 0 .
\end{array}
$$


Let us now discuss the cases $i=i_{p}$, for some $1 \leq p \leq s$. In each of these cases we have obviously $\chi^{\left[i_{1} \ldots i i_{s}\right] I}=0$, being the index $i$ repeated twice. On the other hand, taking into account the antisymmetry of $d s_{i_{1} \ldots i i_{s}}$ in all its indices, and the fact that we have two sums on indices $i$ 's which always must coincide, we can easily check that

$$
\sum_{|I|=0}^{r-1} \mathrm{~d}_{i} \mathrm{~d}_{I} \chi^{i_{1} \ldots\left(i i_{s}\right) i I} \wedge d s_{i_{1} \ldots i i_{s}} \equiv 0 \Longrightarrow \sum_{|I|=0}^{r-1} \mathrm{~d}_{i} \mathrm{~d}_{I} \chi^{i_{1} \ldots i\left(i_{s} i\right) I} \wedge d s_{i_{1} \ldots i i_{s}} \equiv 0
$$

This in turn implies that

$$
\sum_{|I|=0}^{r-1} \mathrm{~d}_{i} \mathrm{~d}_{I} \chi^{i_{1} \ldots i i_{s} i I} \wedge d s_{i_{1} \ldots i i_{s}}=\sum_{|I|=0}^{r-1} \mathrm{~d}_{i} \mathrm{~d}_{I} \chi^{i_{1} \ldots\left[i i_{s}\right] i I} \wedge d s_{i_{1} \ldots i i_{s}},
$$

but this last term is equal (up to a numerical coefficient) to $\sum_{|I|=0}^{r-1} \mathrm{~d}_{i} \mathrm{~d}_{I} \chi^{i_{1} \ldots i_{s}[i] I}$ $\wedge d s_{i_{1} \ldots i i_{s}}$ which is identically vanishing.

Indeed, let $d_{i} \Omega^{i_{1} \ldots i_{s-2} i i_{s} i}:=\sum_{|I|=0}^{r-1} \mathrm{~d}_{i} \mathrm{~d}_{I} \chi^{i_{1} \ldots i i_{s} i I}$. Since $d_{i} \Omega^{\left[i_{1} \ldots i_{s-2} i i_{s} i\right]}=0$ then $d_{i} \Omega^{i_{1} \ldots i_{s-2} i i_{s} i}$ is reduced according to Definition 2.3. On the other hand we also have $d_{i} \Omega^{i_{1} \ldots i_{s-2} i\left(i_{s} i\right)}=0$, thus (as a consequence of [3], Lemma 6.2.43 p. 164) we recover the fact that $d_{i} \Omega^{i_{1} \ldots i_{s-2} i i_{s} i}=d_{i} \Omega^{i_{1} \ldots i_{s-2} i i_{s} i_{s-1}} \equiv 0$. We shall specify this result for $k=1$ in the next Section (see Example 4.2). q.e.d.

Let us now explicate the second summand of equation (12) which will play a fundamental role for the application to Lepage equivalents in Section 5.

Proposition 4.1. Let $\rho \in \Omega_{n-s+k}^{r} W$ be a $(n-s)$-horizontal $k$-contact $(n-$ $s+k)$-form defined on the $r$-order jet prolongation $W^{r}$ of $W$. We have

$$
\begin{aligned}
& d_{i} \sum_{|I|=0}^{r-1} d_{I} \chi^{\left[i_{1} \ldots i_{s}\right] I} \wedge d s_{i_{1} \ldots i_{s}}= \\
& d_{H}\left(\sum_{|I|=0}^{r-1}(-1)^{k} \frac{1}{(s+1)} d_{I} \chi^{\left[i_{1} \ldots i_{s} i\right] I} \wedge d s_{i_{1} \ldots i_{s} i}\right)=d_{H} \mathscr{R}(\rho),
\end{aligned}
$$

where $\mathscr{R}(\rho)$ is a local $(n-s-1)$-horizontal $k$-contact $(n-s-1+k)$-form. 
Geometric integration by parts and Lepage equivalents

Proof. First we rewrite expression (14) using a summation over ordered indices $\tilde{i}_{1} \leq \cdots \leq \tilde{i}_{s}$ instead of $i_{1} \ldots i_{s}$ :

$$
\mathrm{d}_{i} \sum_{|I|=0}^{r-1} \mathrm{~d}_{I} \chi^{\left[i_{1} \ldots i_{s} i\right] I} \wedge d s_{i_{1} \ldots i_{s}}=\mathrm{d}_{i} \sum_{|I|=0}^{r-1} s ! \mathrm{d}_{I} \chi^{\left[\tilde{i}_{1} \ldots \tilde{i}_{s} i\right] I} \wedge d s_{\tilde{i}_{1} \ldots \tilde{i}_{s}} .
$$

Then we expand this sum, using Einstein's convention for the summation over multi-indices $I$ :

$$
\begin{aligned}
& \mathrm{d}_{i} \sum_{|I|=0}^{r-1} s ! \mathrm{d}_{I} \chi^{\left[\tilde{i}_{1} \ldots \tilde{i}_{s} i\right] I} \wedge d s_{\tilde{i}_{1} \ldots \tilde{i}_{s}}= \\
& =s !\left(\mathrm{d}_{1} \mathrm{~d}_{I} \chi^{\left.\left[\tilde{i}_{1} \ldots \tilde{i}_{s}\right]\right] I} \wedge d s_{\tilde{i}_{1} \ldots \tilde{i}_{s}}+\mathrm{d}_{2} \mathrm{~d}_{I} \chi^{\left[\tilde{i}_{1} \ldots \tilde{i}_{s}\right] I I} \wedge d s_{\tilde{i}_{1} \ldots \tilde{i}_{s}}+\ldots\right. \\
& \left.\cdots+\mathrm{d}_{n} \mathrm{~d}_{I} \chi^{\left[\tilde{i}_{1} \ldots \tilde{i}_{s} n\right] I} \wedge d s_{\tilde{i}_{1} \ldots \tilde{i}_{s}}\right) .
\end{aligned}
$$

Let now again write

$$
d s_{\tilde{i}_{1} \ldots \tilde{i}_{s}}=d x^{i} \wedge d s_{\tilde{i}_{1} \ldots \tilde{i}_{s} i}
$$

without summation on the index $i$ and with $i \neq \tilde{i}_{1}, \ldots, \tilde{i}_{s}$. Then we can proceed as follows:

$$
\begin{aligned}
& \mathrm{d}_{i} \sum_{|I|=0}^{r-1} s ! \mathrm{d}_{I} \chi^{\left[\tilde{i}_{1} \ldots \tilde{i}_{s} i\right] I} \wedge d s_{\tilde{i}_{1} \ldots \tilde{i}_{s}}= \\
& =s !\left(\mathrm{d}_{1} \mathrm{~d}_{I} \chi^{\left[\tilde{i}_{1} \ldots \tilde{i}_{s} 1\right] I} \wedge d x^{1} \wedge d s_{\tilde{i}_{1} \ldots \tilde{i}_{s} 1}+\mathrm{d}_{2} \mathrm{~d}_{I} \chi^{\left[\tilde{i}_{1} \ldots \tilde{i}_{s} 2\right] I} \wedge d x^{2} \wedge d s_{\tilde{i}_{1} \ldots \tilde{i}_{s} 2}+\ldots\right. \\
& \left.\cdots+\mathrm{d}_{n} \mathrm{~d}_{I} \chi^{\left[\tilde{i}_{1} \ldots \tilde{i}_{s} n\right] I} \wedge d x^{n} \wedge d s_{\tilde{i}_{1} \ldots \tilde{i}_{s} n}\right) .
\end{aligned}
$$

For each term of the last equation, we have the same indices in $\chi^{\left[\tilde{i}_{1} \ldots \tilde{i}_{s} i\right] I}$ and in $d s_{\tilde{i}_{1} \ldots \tilde{i}_{s} i}$, though without summation on $i$.

Our goal is to take each term $\mathrm{d}_{i} \mathrm{~d}_{I} \chi^{\left[\tilde{i}_{1} \ldots \tilde{i}_{s} i\right] I} \wedge d x^{i} \wedge d s_{\tilde{i}_{1} \ldots \tilde{i}_{s} i}$ without summation on any index and find a way to write it in the form

$$
\mathrm{d}_{l} \mathrm{~d}_{I} \chi^{\left[\tilde{i}_{1} \ldots \tilde{i}_{s} i\right] I} \wedge d x^{l} \wedge d s_{\tilde{i}_{1} \ldots \tilde{i}_{s} i}
$$

with summation on the index $l$. In order to make explicit the reasoning, let us consider the term with $i=1$

$$
\mathrm{d}_{1} \mathrm{~d}_{I} \chi^{\left[\tilde{i}_{1} \ldots \tilde{i}_{s} 1\right] I} \wedge d x^{1} \wedge d s_{\tilde{i}_{1} \ldots \tilde{i}_{s} 1}
$$


again without summation over any index. We have obviously that:

$$
\mathrm{d}_{1} \mathrm{~d}_{I} \chi^{\left.\left[\tilde{i}_{1} \ldots \tilde{i}_{s}\right]\right] I} \wedge d x^{1} \wedge d s_{\tilde{i}_{1} \ldots \tilde{i}_{s} 1}=\sum_{l \neq \tilde{i}_{1}, \ldots, \tilde{i}_{s}} \mathrm{~d}_{l} \mathrm{~d}_{I} \chi^{\left[\tilde{i}_{1} \ldots \tilde{i}_{s} 1\right] I} \wedge d x^{l} \wedge d s_{\tilde{i}_{1} \ldots \tilde{i}_{s} 1} .
$$

The remaining terms when $l=\tilde{i}_{1}, \ldots, l=\tilde{i}_{s}$ can be found among the other summands of the right hand side of equation (15). As an example, consider the case when $l=\tilde{i}_{1}$ : among the terms

$$
\mathrm{d}_{\tilde{i}_{1}} \mathrm{~d}_{I} \chi^{\left[\tilde{j}_{1} \ldots \tilde{j}_{s} \tilde{i}_{1}\right] I} \wedge d x^{\tilde{i}_{1}} \wedge d s_{\tilde{j}_{1} \ldots \tilde{j}_{s} \tilde{i}_{1}}
$$

(with summation on the ordered indices $\tilde{j}_{1} \leq \cdots \leq \tilde{j}_{s}$ ) there will certainly be a summand of the form

$$
\mathrm{d}_{\tilde{i}_{1}} \mathrm{~d}_{I} \chi^{\left[1 \tilde{i}_{2} \ldots \tilde{i}_{s} \tilde{i}_{1}\right] I} \wedge d x^{\tilde{i}_{1}} \wedge d s_{1 \tilde{i}_{2} \ldots \tilde{i}_{s} \tilde{i}_{1}}
$$

(this time without summation on any index) which can be recast as

$$
\mathrm{d}_{\tilde{i}_{1}} \mathrm{~d}_{I} \chi^{\left[\tilde{i}_{1} \ldots \tilde{i}_{s} 1\right] I} \wedge d x^{\tilde{i}_{1}} \wedge d s_{\tilde{i}_{1} \ldots \tilde{s}_{s} 1} .
$$

This is exactly the term we were searching for. Proceeding in the same way for $l=\tilde{i}_{2}, \ldots, l=\tilde{i}_{s}$, we can finally obtain the expression

$$
\mathrm{d}_{l} \mathrm{~d}_{I} \chi^{\left[\tilde{i}_{1} \ldots \tilde{i}_{s} 1\right] I} \wedge d x^{l} \wedge d s_{\tilde{i}_{1} \ldots \tilde{i}_{s} 1}
$$

(with summation on $l$ ) as wanted. Moreover, we remark that the total number of summands of equation (15) is $N=n\left(\begin{array}{c}n-1 \\ s\end{array}\right)$, while the number of ordered strings of $(s+1)$ indices is $N^{\prime}=\left(\begin{array}{c}n \\ s+1\end{array}\right)$, and $\stackrel{s}{N}=(s+1) N^{\prime}$. This means that for every single term of equation (15) $\mathrm{d}_{i} \mathrm{~d}_{I} \chi^{\left[\tilde{1}_{1} \ldots \tilde{s}_{s} i\right] I} \wedge d x^{i} \wedge d s_{\tilde{i}_{1} \ldots \tilde{i}_{s} i}$ (without summation on any index), there are other $s$ terms with the same indices, just in a different order.

Therefore, it is not difficult to see that

$$
\mathrm{d}_{i} \sum_{|I|=0}^{r-1} s ! \mathrm{d}_{I} \chi^{\left[\tilde{i}_{1} \ldots \tilde{s}_{s} i\right] I} \wedge d s_{\tilde{i}_{1} \ldots \tilde{i}_{s}}=\mathrm{d}_{l} \sum_{|I|=0}^{r-1} s ! \mathrm{d}_{I} \chi^{\left[\tilde{i}_{1} \ldots \tilde{i}_{s} \tilde{s}_{s+1}\right] I} \wedge d x^{l} \wedge d s_{\tilde{i}_{1} \ldots \tilde{i}_{s} \tilde{s}_{s+1}}
$$

with summation on ordered indices $\tilde{i}_{1} \leq \cdots \leq \tilde{i}_{s} \leq \tilde{i}_{s+1}$. Passing to a summation on non-ordered indices $i_{1} \ldots i_{s+1}$ and using commutation properties 
Geometric integration by parts and Lepage equivalents

of wedge products, we can finally write:

$$
\begin{aligned}
& \mathrm{d}_{i} \sum_{|I|=0}^{r-1} \mathrm{~d}_{I} \chi^{\left[i_{1} \ldots i_{s} i\right] I} \wedge d s_{i_{1} \ldots i_{s}}= \\
& =\mathrm{d}_{l} \sum_{|I|=0}^{r-1} \frac{1}{(s+1)} \mathrm{d}_{I} \chi^{\left[i_{1} \ldots i_{s+1}\right] I} \wedge d x^{l} \wedge d s_{i_{1} \ldots i_{s+1}}= \\
& =(-1)^{n-s-1+k} \mathrm{~d}_{l}\left(\sum_{|I|=0}^{r-1}(-1)^{k} \frac{1}{(s+1)} \mathrm{d}_{I} \chi^{\left[i_{1} \ldots i_{s+1}\right] I} \wedge d s_{i_{1} \ldots i_{s+1}}\right) \wedge d x^{l}= \\
& =d_{H}\left(\sum_{|I|=0}^{r-1}(-1)^{k} \frac{1}{(s+1)} \mathrm{d}_{I} \chi^{\left[i_{1} \ldots i_{s+1}\right] I} \wedge d s_{i_{1} \ldots i_{s+1}}\right):=d_{H} \mathscr{R}(\rho) .
\end{aligned}
$$

q.e.d.

Definition 4.1. Let $\rho \in \Omega_{n-s+k}^{r} W$ be a $(n-s)$-horizontal $k$-contact $(n-$ $s+k)$-form and let $p_{k} \rho=\sum_{|J|=0}^{r} \omega_{J}^{\sigma} \wedge \eta_{\sigma}^{J} \in \Omega_{n-s+k}^{r+1} W$.

We define the Residual operator associated with $\rho$ as the operator $\mathscr{R}$ locally characterized by the above Proposition where the forms $\chi$ 's are defined in terms of the forms $\eta$ 's by equations (10) and (11).

\subsection{Comparison in the case $k=1$}

In order to compare the results above with the canonical splitting of variational morphisms, let us restrict to the case $k=1$.

Proposition 4.2. Let $p_{1} \rho=\sum_{|L|=0}^{r} \omega_{L}^{\sigma} \wedge \eta_{\sigma}^{L} \in \Omega_{n-s+1}^{r+1} W$, with

$$
\eta_{\sigma}^{L}=A_{\sigma}^{i_{1} \ldots i_{s} L} \wedge d s_{i_{1} \ldots i_{s}}
$$

where the coefficients $A_{\sigma}^{i_{1} \ldots i_{s} L}$ are defined on $J^{r+1} W$. We have a "canonical splitting"-like decomposition

$$
\begin{gathered}
p_{1} \rho=\sum_{k=0}^{r}(-1)^{k} d_{a_{k} \ldots a_{3} a_{2} a_{1}} A_{\sigma}^{\left[i_{1} \ldots i_{s} a_{1}\right] a_{2} a_{3} \ldots a_{k}} \omega^{\sigma} \wedge d s_{i_{1} \ldots i_{s}}+ \\
+d_{H}\left[\frac{1}{(s+1)} \sum_{|L|=0}^{r-1}-\hat{t}_{\sigma}^{i_{1} \ldots i_{s} i L} \omega_{L}^{\sigma} \wedge d s_{i_{1} \ldots i_{s} i}\right]
\end{gathered}
$$


Geometric integration by parts and Lepage equivalents

where the coefficients $\hat{t}$ 's are defined iteratively by:

$$
\begin{aligned}
& \hat{t}_{\sigma}^{i_{1} \ldots i_{s} i l_{1} \ldots l_{r-1}}=A_{\sigma}^{\left.\left[i_{1} \ldots i_{s}\right]\right]_{1} \ldots l_{r-1}}
\end{aligned}
$$

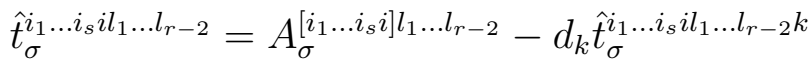

$$
\begin{aligned}
& \text {... } \\
& \hat{t}_{\sigma}^{i_{1} \ldots i_{s} i}=A_{\sigma}^{\left[i_{1} \ldots i_{s} i\right]}-d_{k} \hat{t}_{\sigma}^{i_{1} \ldots i_{s} i k} .
\end{aligned}
$$

Proof. We have

$$
\xi_{\sigma}^{I}=\left(\sum_{|J|=0}^{r-|I|}(-1)^{|J|}\left(\begin{array}{c}
|J|+|I| \\
|J|
\end{array}\right) d_{J} A_{\sigma}^{i_{1} \ldots i_{s} I J}\right) d s_{i_{1} \ldots i_{s}}
$$

and consequently, in the case $k=1$, we put

$$
\chi^{i_{1} \ldots i_{s} I}=\sum_{|J|=0}^{r-|I|}(-1)^{|J|}\left(\begin{array}{c}
|J|+|I| \\
|J|
\end{array}\right) d_{J} A_{\sigma}^{i_{1} \ldots i_{s} I J} \omega^{\sigma},
$$

therefore Proposition 4.1 specializes as

$$
\begin{aligned}
& \mathrm{d}_{i} \sum_{|I|=0}^{r-1} \mathrm{~d}_{I} \chi^{\left[i_{1} \ldots i_{s} i\right] I} \wedge d s_{i_{1} \ldots i_{s}}= \\
& =d_{H}\left[\sum_{|I|=0}^{r-1} \frac{-1}{(s+1)} \mathrm{d}_{I} \chi^{\left[i_{1} \ldots i_{s} i\right] I} \wedge d s_{i_{1} \ldots i_{s} i}\right]= \\
& =d_{H}\left[\frac{-1}{(s+1)} \sum_{|I|=0}^{r-1} \mathrm{~d}_{I}\left(\sum_{|J|=0}^{r-|i I|}(-1)^{|J|}\left(\begin{array}{c}
|J|+|i I| \\
|J|
\end{array}\right) d_{J} A_{\sigma}^{\left[i_{1} \ldots i_{s} i\right] I J} \omega^{\sigma}\right) \wedge d s_{i_{1} \ldots i_{s}}\right] .
\end{aligned}
$$

The explicit expression of this term can be computed as described in Step 2 of the proof of Proposition 3.1. In particular, if we develop the total derivatives $\mathrm{d}_{I}$ inside the sum in equation (19) and collect the coefficients of the contact forms of the same order, we obtain the expression

$$
\begin{aligned}
& d_{H}\left[\frac{-1}{(s+1)} \sum_{|I|=0}^{r-1} \mathrm{~d}_{I}\left(\sum_{|J|=0}^{r-|i I|}(-1)^{|J|}\left(\begin{array}{c}
|J|+|i I| \\
|J|
\end{array}\right) d J A_{\sigma}^{\left[i_{1} \ldots i_{s} i\right] I J} \omega^{\sigma}\right) \wedge d s_{i_{1} \ldots i_{s} i}\right]= \\
& =d_{H}\left[-\frac{1}{(s+1)} \sum_{|L|=0}^{r-1} \hat{t}_{\sigma}^{i_{1} \ldots i_{s} i L} \omega_{L}^{\sigma} \wedge d s_{i_{1} \ldots i_{s} i}\right] .
\end{aligned}
$$


Because of its role in the extension of the interior Euler operator to the cases of lower degree, we now proceed in particular to determine the coefficient of $\omega^{\sigma} \wedge d s_{i_{1} \ldots i_{s}}$. The first term we need to consider is obviously

$$
A_{\sigma}^{i_{1} \ldots i_{s}} \omega^{\sigma} \wedge d s_{i_{1} \ldots i_{s}} .
$$

Put $B_{\sigma}^{i_{1} \ldots i_{s} i I J}=A_{\sigma}^{i_{1} \ldots i_{s} i I J}-A_{\sigma}^{\left[i_{1} \ldots i_{s} i\right] I J}$. The other contributions then, of course, come from

$$
\sum_{|I|=0}^{r-1} \mathrm{~d}_{i} \mathrm{~d}_{I}\left(\sum_{|J|=0}^{r-|i I|}(-1)^{|J|}\left(\begin{array}{c}
|J|+|i I| \\
|J|
\end{array}\right) d_{J} B_{\sigma}^{i_{1} \ldots i_{s} i I J}\right) \omega^{\sigma} \wedge d s_{i_{1} \ldots i_{s}} .
$$

In other words, renaming $i I \rightarrow I$, we compute the expression:

$$
\sum_{|J|=0}^{r}(-1)^{|J|} d_{J} A_{\sigma}^{i_{1} \ldots i_{s} J}+\sum_{|I|=1}^{r} \sum_{|J|=0}^{r-|I|}(-1)^{|J|}\left(\begin{array}{c}
|J|+|I| \\
|J|
\end{array}\right) d_{I} d_{J} B_{\sigma}^{i_{1} \ldots i_{s} I J} .
$$

We collect the terms according to the length $|L|$ of the multi-index $J$ in the first sum and $I J$ in the second sum:

$|L|=0$ Since the second double sum starts with $|I|=1$, we have only the first term $A_{\sigma}^{i_{1} \ldots i_{s}}$ from the first sum.

$|L|=1$ From the first sum we get the term $-d_{i} A_{\sigma}^{i_{1} \ldots i_{s} i}$, while from the second sum, considering $|I|=1$ and $|J|=0$, we get $d_{i} B_{\sigma}^{i_{1} \ldots i_{s} i}$. Summing up these contribution we obtain:

$$
-d_{i} A_{\sigma}^{i_{1} \ldots i_{s} i}+d_{i} B_{\sigma}^{i_{1} \ldots i_{s} i}=-d_{i} A_{\sigma}^{\left[i_{1} \ldots i_{s} i\right]} .
$$

$|L|=2$ From the first sum we get the term $d_{a b} A_{\sigma}^{i_{1} \ldots i_{s} a b}$, while in the second sum we can choose $|I|=2$ and $|J|=0$, getting the term $d_{a b} B_{\sigma}^{i_{1} \ldots i_{s} a b}$, or $|I|=1$ and $|J|=1$, getting the term $-2 d_{a b} B_{\sigma}^{i_{1} \ldots i_{s} a b}$. Summing all together, we obtain:

$$
d_{a b} A_{\sigma}^{i_{1} \ldots i_{s} a b}-d_{a b} B_{\sigma}^{i_{1} \ldots i_{s} a b}=d_{a} d_{b} A_{\sigma}^{\left[i_{1} \ldots i_{s} a\right] b} .
$$

It is not difficult to see that, for general length $|L|$, we obtain the term:

$$
\begin{aligned}
& (-1)^{|L|} d_{a_{1} \ldots a_{|L|}} A_{\sigma}^{i_{1} \ldots i_{s} a_{1} \ldots a_{|L|}}+\sum_{k=0}^{|L|-1}(-1)^{k}\left(\begin{array}{c}
|L| \\
k
\end{array}\right) d_{a_{1} \ldots a_{|L|}} B_{\sigma}^{i_{1} \ldots i_{s} a_{1} \ldots a_{|L|}}= \\
& =(-1)^{|L|}\left(d_{a_{1} \ldots a_{|L|}} A_{\sigma}^{i_{1} \ldots i_{s} a_{1} \ldots a_{|L|}}-d_{a_{1} \ldots a_{|L|}} B_{\sigma}^{i_{1} \ldots i_{s} a_{1} \ldots a_{|L|}}\right)= \\
& =(-1)^{|L|} d_{a_{1} \ldots a_{|L|}} A_{\sigma}^{\left[i_{1} \ldots i_{s} a_{1}\right] a_{2} \ldots a_{|L|}} .
\end{aligned}
$$


Geometric integration by parts and Lepage equivalents

where we used $\sum_{k=0}^{|L|-1}(-1)^{k}\left(\begin{array}{c}|L| \\ k\end{array}\right)=-(-1)^{|L|}$. Thence finally, the coefficient of the form $\omega^{\sigma} \wedge d s_{i_{1} \ldots i_{s}}$ is given by:

$$
\begin{array}{r}
A_{\sigma}^{i_{1} \ldots i_{s}}-d_{a_{1}} A_{\sigma}^{\left[i_{1} \ldots i_{s} a_{1}\right]}+d_{a_{1}} d_{a_{2}} A_{\sigma}^{\left[i_{1} \ldots i_{s} a_{1}\right] a_{2}}+\ldots \\
\cdots+(-1)^{r} d_{a_{1}} \ldots d_{a_{r}} A_{\sigma}^{\left[i_{1} \ldots i_{s} a_{1}\right] a_{2} \ldots a_{r}}
\end{array}
$$

We now study the terms generated by $\omega_{L}^{\sigma} \wedge d s_{i_{1} \ldots i_{s}}$. We explicate the total derivatives of forms in the following expression

$$
\begin{aligned}
& \sum_{|I|=0}^{r-1} \mathrm{~d}_{i} \mathrm{~d}_{I}\left(\chi^{i_{1} \ldots i_{s} i I}-\chi^{\left[i_{1} \ldots i_{s} i\right] I}\right) \wedge d s_{i_{1} \ldots i_{s}}= \\
& =\sum_{|I|=0}^{r-1} \mathrm{~d}_{i} \mathrm{~d}_{I}\left(\sum_{|J|=0}^{r-|i I|}(-1)^{|J|}\left(\begin{array}{c}
|J|+|i I| \\
|J|
\end{array}\right) d_{J} B_{\sigma}^{i_{1} \ldots i_{s} i I J} \omega^{\sigma}\right) \wedge d s_{i_{1} \ldots i_{s}}
\end{aligned}
$$

and get the coefficients of these terms according to the length of $L$.

$|L|=r$ The only contribution to this coefficient comes from setting $|I|=r-1$ in equation (23) and applying all the total derivatives to $\omega^{\sigma}$. Renaming the multi-index $L=l L^{\prime}$ (hence with $\left|L^{\prime}\right|=|L|-1$ ), we obtain:

$$
B_{\sigma}^{i_{1} \ldots i_{s} l L^{\prime}}=A_{\sigma}^{i_{1} \ldots i_{s} l L^{\prime}}-A_{\sigma}^{\left[i_{1} \ldots i_{s} l\right] L^{\prime}} .
$$

$|L|=r-1$ One first contribution comes from setting $|I|=r-2$ and applying all the total derivatives to $\omega^{\sigma}$, getting:

$$
B_{\sigma}^{i_{1} \ldots i_{s} L}-r d_{i} B_{\sigma}^{i_{1} \ldots i_{s} L i} .
$$

Another contribution comes from setting $|I|=r-1$ and applying one derivative to $B$ and the others to $\omega^{\sigma}$, getting:

$$
d_{i} B_{\sigma}^{i_{1} \ldots i_{s} i L}+\left(\begin{array}{c}
r-1 \\
1
\end{array}\right) d_{i} B_{\sigma}^{i_{1} \ldots i_{s} L i}
$$

Summing up these contributions, and renaming $L=l L^{\prime}$, we obtain:

$$
\begin{aligned}
& B_{\sigma}^{i_{1} \ldots i_{s} l L^{\prime}}-d_{i} B_{\sigma}^{i_{1} \ldots i_{s} l L^{\prime} i}+d_{i} B_{\sigma}^{i_{1} \ldots i_{s} i l L^{\prime}}= \\
& =A_{\sigma}^{i_{1} \ldots i_{s} l L^{\prime}}-A_{\sigma}^{\left[i_{1} \ldots i_{s} l\right] L^{\prime}}-d_{i} A_{\sigma}^{i_{1} \ldots i_{s} l L^{\prime} i}+d_{i} A_{\sigma}^{\left[i_{1} \ldots i_{s} l\right] L^{\prime} i}+ \\
& +d_{i} A_{\sigma}^{i_{1} \ldots i_{s} i l L^{\prime}}-d_{i} A_{\sigma}^{\left.\left[i_{1} \ldots i_{s}\right]\right] l L^{\prime}}= \\
& =A_{\sigma}^{i_{1} \ldots i_{s} l L^{\prime}}-A_{\sigma}^{\left[i_{1} \ldots i_{s} l\right] L^{\prime}}+d_{i} A_{\sigma}^{\left[i_{1} \ldots i_{s} l\right] L^{\prime} i}-d_{i} A_{\sigma}^{\left.\left[i_{1} \ldots i_{s}\right]\right] l L^{\prime}} .
\end{aligned}
$$


Geometric integration by parts and Lepage equivalents

$|L|=r-2$ One contribution comes from setting $|I|=r-3$ and applying all the derivatives to $\omega^{\sigma}$. Another contribution comes from setting $|I|=$ $r-2$ and applying only $r-2$ derivatives to $\omega^{\sigma}$. One last contribution comes from setting $|I|=r-1$ and applying only $r-2$ derivatives to $\omega^{\sigma}$. Summing up, the coefficient for $|L|=r-2$ is:

$$
\begin{gathered}
A_{\sigma}^{i_{1} \ldots i_{s} l L^{\prime}}-A_{\sigma}^{\left[i_{1} \ldots i_{s} l\right] L^{\prime}}+d_{i} A_{\sigma}^{\left.\left[i_{1} \ldots i_{s}\right]\right] L^{\prime} i}-d_{i} A_{\sigma}^{\left[i_{1} \ldots i_{s} i\right] l L^{\prime}} \\
-d_{a i} A_{\sigma}^{\left[i_{1} \ldots i_{s} l\right] L^{\prime} a i}+d_{i a} A_{\sigma}^{\left[i_{1} \ldots i_{s} i\right] a l L^{\prime}} .
\end{gathered}
$$

$|L|=r-g, 0 \leq g \leq r$ in general we have

$$
\sum_{m=0}^{g}(-1)^{m} d_{j_{m} \ldots j_{1}}\left(A_{\sigma}^{\left[i_{1} \ldots i_{s} l\right] L^{\prime} j_{m} \ldots j_{1}}-A_{\sigma}^{\left[i_{1} \ldots i_{s} j_{m}\right] j_{m-1} \ldots j_{1} l L^{\prime}}\right) \omega_{l L^{\prime}}^{\sigma} \wedge d s_{i_{1} \ldots i_{s}} .
$$

Note that we recover expressions (22) for $|L|=\left|l L^{\prime}\right|=0$.

Integrating by parts we obtain

$$
\begin{aligned}
& \sum_{m=0}^{g}(-1)^{m} d_{l}\left(d_{j_{m} \ldots j_{1}}\left(A_{\sigma}^{\left[i_{1} \ldots i_{s} l\right] L^{\prime} j_{m} \ldots j_{1}}-A_{\sigma}^{\left[i_{1} \ldots i_{s} j_{m}\right] j_{m-1} \ldots j_{1} l L^{\prime}}\right) \omega_{L^{\prime}}^{\sigma}\right) \wedge d s_{i_{1} \ldots i_{s}}+ \\
& -\sum_{m=0}^{g}(-1)^{m} d_{l}\left(d_{j_{m} \ldots j_{1}}\left(A_{\sigma}^{\left[i_{1} \ldots i_{s}\right]\left[L^{\prime} j_{m} \ldots j_{1}\right.}-A_{\sigma}^{\left[i_{1} \ldots i_{s} j_{m}\right] j_{m-1} \ldots j_{1} l L^{\prime}}\right)\right) \omega_{L^{\prime}}^{\sigma} \wedge d s_{i_{1} \ldots i_{s}}
\end{aligned}
$$

where the last piece (apart the case $g=0$, contributing the coefficient of $\left.\omega_{L^{\prime}}^{\sigma} \wedge d s_{i_{1} \ldots i_{s}}\right)$ is zero because of the symmetry in the indices $l$ and $j_{m}$.

For $g \neq 0$, and any choice of $l=j_{p} 1 \leq p \leq r$, taking into account the symmetry of the indices $j_{m} \ldots j_{p} \ldots j_{3} j_{2} j_{1}$, the two summands of the first piece become

$$
d_{j_{p}} d_{j_{m} \ldots j_{p} \ldots j_{3} j_{2} j_{1}}\left(A_{\sigma}^{\left[i_{1} \ldots i_{s} j_{p}\right] L^{\prime} j_{m} \ldots j_{2} j_{1}}-A_{\sigma}^{\left[i_{1} \ldots i_{s} j_{m}\right] j_{m-1} \ldots j_{2} j_{1} j_{p} L^{\prime}}\right) \equiv 0 .
$$

For $g=0$ and $l \neq i_{p}$

$$
\begin{array}{r}
d_{l}\left(\left(A_{\sigma}^{\left[i_{1} \ldots i_{s} l\right] L^{\prime}}-A_{\sigma}^{\left[i_{1} \ldots i_{s}\right] l L^{\prime}}\right) \omega_{L^{\prime}}^{\sigma}\right) \wedge d s_{i_{1} \ldots i_{s}}= \\
d_{l}\left(\left(A_{\sigma}^{\left.\left[i_{1} \ldots\left[i_{s}\right]\right]\right] L^{\prime}}-A_{\sigma}^{\left[i_{1} \ldots\left[i_{s}\right] l\right] L^{\prime}}\right) \omega_{L^{\prime}}^{\sigma}\right) \wedge d x^{l} \wedge d s_{i_{1} \ldots i_{s} l}=0 .
\end{array}
$$

For the case $l=i_{p}$, for some $p$, we refer to the discussion in the general case $g \geq 1$, Lemma 4.1 ,

q.e.d. 
Geometric integration by parts and Lepage equivalents

Definition 4.2. Expression (22) defines a local interior Euler operator for lower degree 1-contact forms

$$
\begin{array}{r}
\mathfrak{I}(\rho)=\omega^{\sigma} \wedge\left(A_{\sigma}^{i_{1} \ldots i_{s}}-d_{a_{1}} A_{\sigma}^{\left[i_{1} \ldots i_{s} a_{1}\right]}+d_{a_{1}} d_{a_{2}} A_{\sigma}^{\left[i_{1} \ldots i_{s} a_{1}\right] a_{2}}+\ldots\right. \\
\left.\cdots+(-1)^{r} d_{a_{1}} \ldots d_{a_{r}} A_{\sigma}^{\left[i_{1} \ldots i_{s} a_{1}\right] a_{2} \ldots a_{r}}\right) d s_{i_{1} \ldots i_{s}} .
\end{array}
$$

It is an expression analogous to (8) and the study of its uniqueness and globality properties will be done elsewhere.

Remark 4.1. For the cases $s=1$ and $k \geq 1$, the splitting $p_{1} \rho=\omega^{\sigma} \wedge$ $\xi_{\sigma}+d_{H}\left(\sum_{|I|=0}^{r-1}-\frac{1}{(s+1)} \mathrm{d}_{I} \chi^{i_{1} \ldots i_{s} i I} \wedge d s_{i_{1} \ldots i_{s} i}\right)$ first appeared in [16], however the explicit local expressions for $\xi_{\sigma}$ are obtained here for the first time (for $k=1$ ). Furthermore, a Residual operator is here locally characterized for any $s \geq 1$.

Remark 4.2. Let us consider $p_{1} \rho \in \Omega_{n-s+1}^{r+1} W$ as a variational morphism

$$
\mathbb{V}_{\rho}: J^{r+1} W \longrightarrow\left(J^{r} V(W)\right)^{*} \otimes A_{n-s}(U)
$$

with $\left.<\mathbb{V}_{\rho} \mid J^{r} \Xi>:=J^{r} \Xi\right\lrcorner p_{1} \rho=\left(A_{\sigma}^{i_{1} \ldots i_{s}} \Xi^{\sigma}+\cdots+A_{\sigma}^{i_{1} \ldots i_{s} j_{1} \ldots j_{r}} \Xi_{j_{1} \ldots j_{r}}^{\sigma}\right) \wedge d s_{i_{1} \ldots i_{s}}$. On the other hand, according to Proposition 4.2, with $p_{1} \rho \in \Omega_{n-s+1}^{r+1} W$ we also associate two variational morphisms

$$
\begin{aligned}
& \mathbb{E}^{\prime}: J^{2 r+1} W \longrightarrow\left(J^{r} V(W)\right)^{*} \otimes A_{n-s}(U) \\
& \mathbb{T}^{\prime}: J^{2 r} W \longrightarrow\left(J^{r-1} V(W)\right)^{*} \otimes A_{n-s-1}(U),
\end{aligned}
$$

such that $<\mathbb{V}_{\rho}\left|J^{r} \Xi>=<\mathbb{E}^{\prime}\right| J^{r} \Xi>+\operatorname{Div}\left(<\mathbb{T}^{\prime} \mid J^{r-1} \Xi>\right)$ and in particular we have the correspondences: $\left.<\mathbb{E}^{\prime} \mid J^{r} \Xi>=J^{r} \Xi\right\lrcorner\left(\omega^{\sigma} \wedge \xi_{\sigma}\right)$, $\operatorname{Div}(<$ $\left.\left.\mathbb{T}^{\prime} \mid J^{r-1} \Xi>\right)=J^{r} \Xi\right\lrcorner d_{H}\left(\sum_{|I|=0}^{r-1}-\frac{1}{(s+1)} \mathrm{d}_{I} \chi^{\left[i_{1} \ldots i_{s} i\right] I} \wedge d s_{i_{1} \ldots i_{s} i}\right)$.

By equations (20) and (17)

$$
<\mathbb{T}^{\prime} \mid J^{r-1} \Xi>=\left(\hat{t}_{\sigma}^{i_{1} \ldots i_{s+1}} \Xi^{\sigma}+\cdots+\hat{t}_{\sigma}^{i_{1} \ldots i_{s+1} j_{1} \ldots j_{r-1}} \Xi_{j_{1} \ldots j_{r-1}}^{\sigma}\right) \wedge d s_{i_{1} \ldots i_{s} i_{s+1}},
$$

while

$$
<\mathbb{E}^{\prime} \mid J^{r} \Xi>=\left(\hat{e}_{\sigma}^{i_{1} \ldots i_{s}} \Xi^{\sigma}+\hat{e}_{\sigma}^{i_{1} \ldots i_{s} j_{1}} \Xi_{j_{1}}^{\sigma}+\cdots+\hat{e}_{\sigma}^{i_{1} \ldots i_{s} j_{1} \ldots j_{r}} \Xi_{j_{1} \ldots j_{r}}^{\sigma}\right) \wedge d s_{i_{1} \ldots i_{s}}
$$

and the general form of the coefficients $\hat{e}$ are given by Proposition 4.2, they can be compared with the canonical splitting morphisms according to [3].

In view of possible applications to momentum morphisms, i.e. 1-contact $(n-1)$-forms (related to the Poincaré-Cartan morphism), we make now some explicit examples for the cases $r=1,2$. 
Example 4.1. Let $r=1,0 \leq s<n$ and let $\rho \in \Omega_{n-s+1}^{1} W$ be a 1-contact $(n-s)$-horizontal form on $W^{1}$. Then the two splitting formulae:

$<\mathbb{V}_{\rho}\left|J^{1} \Xi>=<\mathbb{E}\right| J^{1} \Xi>+\operatorname{Div}(<\mathbb{T} \mid \Xi>)=<\mathbb{E}^{\prime} \mid J^{1} \Xi>+\operatorname{Div}\left(<\mathbb{T}^{\prime} \mid \Xi>\right)$

give the same result. In other words:

$$
\mathbb{E}^{\prime}=\mathbb{E}, \quad \mathbb{T}^{\prime}=\mathbb{T}
$$

Indeed, we observe that locally we have

$$
p_{1} \rho=\left(A_{\sigma}^{i_{1} \ldots i_{s}} \omega^{\sigma}+A_{\sigma}^{i_{1} \ldots i_{s} j} \omega_{j}^{\sigma}\right) \wedge d s_{i_{1} \ldots i_{s}} \in \Omega_{n-s+1}^{2} W .
$$

Thence, for any vertical vector field $\Xi: W \longrightarrow V(W)$, the variational morphism $\mathbb{V}_{\rho}: J^{2} W \longrightarrow\left(J^{1} V(W)\right)^{*} \otimes A_{n-s}(U)$ is defined by:

$$
<\mathbb{V}_{\rho} \mid J^{1} \Xi>=\frac{1}{s !}\left(s ! A_{\sigma}^{i_{1} \ldots i_{s}} \Xi^{\sigma}+s ! A_{\sigma}^{i_{1} \ldots i_{s} j} \Xi_{j}^{\sigma}\right) \wedge d s_{i_{1} \ldots i_{s}} .
$$

Applying to this expression of $\mathbb{V}_{\rho}$ the canonical splitting algorithm from the theory of variational morphisms (see [3]), we have the following volume term and boundary term, respectively, which we compare with our 'canonical splitting'-like decomposition (16):

$$
\begin{aligned}
& \mathbb{E}=\frac{1}{s !}\left[s !\left(A_{\sigma}^{i_{1} \ldots i_{s}}-d_{k} A_{\sigma}^{\left[i_{1} \ldots i_{s} k\right]}\right) \omega^{\sigma}+s !\left(A_{\sigma}^{i_{1} \ldots i_{s} j}-A_{\sigma}^{\left[i_{1} \ldots i_{s} j\right]}\right) \omega_{j}^{\sigma}\right] \wedge d s_{i_{1} \ldots i_{s}} \\
& =\mathbb{E}^{\prime}+\left(A_{\sigma}^{i_{1} \ldots i_{s} j}-A_{\sigma}^{\left[i_{1} \ldots i_{s} j\right]}\right) \omega_{j}^{\sigma} \wedge d s_{i_{1} \ldots i_{s}} .
\end{aligned}
$$

However, according to Proposition $4.2,\left(A_{\sigma}^{i_{1} \ldots i_{s} j}-A_{\sigma}^{\left[i_{1} \ldots i_{s} j\right]}\right) \omega_{j}^{\sigma} \wedge d s_{i_{1} \ldots i_{s}} \equiv 0$. Furthermore,

$$
\mathbb{T}=\frac{1}{(s+1) !}\left[s ! A_{\sigma}^{\left[i_{1} \ldots i_{s} i\right]} \omega^{\sigma}\right] \wedge d s_{i_{1} \ldots i_{s} i}=\mathbb{T}^{\prime} .
$$

Example 4.2. Let $r=2$ and $s=1$ and let $\rho \in \Omega_{n}^{2} W$ be a 1-contact $(n-1)$ horizontal form on $W^{2}$. Then there exists a variational morphism

$$
\alpha: J^{4} W \longrightarrow\left(J^{1} V(W)\right)^{*} \otimes A_{n-2}(U)
$$

such that the decompositions

$$
<\mathbb{V}_{\rho}\left|J^{2} \Xi>=<\mathbb{E}\right| J^{2} \Xi>+\operatorname{Div}\left(<\mathbb{T} \mid J^{1} \Xi>\right)=<\mathbb{E}^{\prime} \mid J^{2} \Xi>+\operatorname{Div}\left(<\mathbb{T}^{\prime} \mid J^{1} \Xi>\right)
$$


are related by

$$
\mathbb{E}^{\prime}=\mathbb{E}-\mathcal{D} \alpha, \quad \mathbb{T}^{\prime}=\mathbb{T}+\alpha,
$$

where $\mathcal{D} \alpha: J^{5} W \longrightarrow\left(J^{2} V(W)\right)^{*} \otimes A_{n-1}(U)$ is the unique variational morphism such that $<\mathcal{D} \alpha \mid J^{2} \Xi>=\operatorname{Div}\left(<\alpha \mid J^{1} \Xi>\right)$.

In fact, let $p_{1} \rho=\left(A_{\sigma}^{i} \omega^{\sigma}+A_{\sigma}^{i j_{1}} \omega_{j_{1}}^{\sigma}+A_{\sigma}^{i j_{1} j_{2}} \omega_{j_{1} j_{2}}^{\sigma}\right) \wedge d s_{i} \in \Omega_{n}^{3} W$. Choosing the fibered connection whose coefficients vanish in the coordinate domain $W \subset Y$,

$$
\begin{aligned}
& <\mathbb{E} \mid J^{2} \Xi>=\left[\left(A_{\sigma}^{i}-d_{a} A_{\sigma}^{[i a]}-\frac{2}{3} d_{b} d_{a} A_{\sigma}^{[i b] a}\right) \Xi^{\sigma}+\right. \\
& \left.+\left(A_{\sigma}^{\left(i j_{1}\right)}+\frac{2}{3} d_{a} A_{\sigma}^{a i j_{1}}-\frac{2}{3} d_{a} A_{\sigma}^{\left(i j_{1}\right) a}\right) \Xi_{j_{1}}^{\sigma}+A_{\sigma}^{\left(i j_{1} j_{2}\right)} \Xi_{j_{1} j_{2}}^{\sigma}\right] \wedge d s_{i} .
\end{aligned}
$$

After some manipulations

$$
\begin{aligned}
& \mathbb{E}=\mathbb{E}^{\prime}+\left[\left(A_{\sigma}^{i j_{1}}-d_{a} A_{\sigma}^{[i a] j_{1}}-A_{\sigma}^{\left[i j_{1}\right]}+d_{a} A_{\sigma}^{\left[i j_{1}\right] a}\right) \omega_{j_{1}}^{\sigma}+\right. \\
& \left.+\left(A_{\sigma}^{i j_{1} j_{2}}-A_{\sigma}^{\left[i j_{1}\right] j_{2}}\right) \omega_{j_{1} j_{2}}^{\sigma}\right] \wedge d s_{i}+\mathcal{D}(\alpha) .
\end{aligned}
$$

where

$\mathcal{D}(\alpha)=-\left[\frac{1}{3} d_{b} d_{a} A_{\sigma}^{[i b] a} \omega^{\sigma}+\left(\frac{1}{3} d_{a} A_{\sigma}^{\left[i j_{1}\right] a}+\frac{1}{3} d_{a} A_{\sigma}^{[i a] j_{1}}\right) \omega_{j_{1}}^{\sigma}+\frac{1}{3} A_{\sigma}^{\left[i j_{1}\right] j_{2}} \omega_{j_{1} j_{2}}^{\sigma}\right] \wedge d s_{i}$, and one can check that the second summand of (26) vanishes.

In order to compute $\mathbb{T}^{\prime}$ from equation (19), let $\chi^{i j_{1}}=\left(A_{\sigma}^{i j_{1}}-2 d_{a} A_{\sigma}^{i j_{1} a}\right) \omega^{\sigma}$, $\chi^{i j_{1} j_{2}}=A_{\sigma}^{i j_{1} j_{2}} \omega^{\sigma}$, then

$$
\begin{aligned}
& \mathbb{T}^{\prime}=\sum_{|J|=0}^{1} \frac{1}{2} d_{J} \chi^{\left[i_{1} i_{2}\right] J} \wedge d s_{i_{1} i_{2}}=\frac{1}{2} \chi^{\left[i_{1} i_{2}\right]} \wedge d s_{i_{1} i_{2}}+\frac{1}{2} d_{a} \chi^{\left[i_{1} i_{2}\right] a} \wedge d s_{i_{1} i_{2}}= \\
& =\left[\frac{1}{2}\left(A_{\sigma}^{\left[i_{1} i_{2}\right]}-d_{a} A_{\sigma}^{\left[i_{1} i_{2}\right] a}\right) \omega^{\sigma}+\frac{1}{2} A_{\sigma}^{\left[i_{1} i_{2}\right] a} \omega_{a}^{\sigma}\right] \wedge d s_{i_{1} i_{2}} .
\end{aligned}
$$

Comparing with

$$
<\mathbb{T} \mid J^{1} \Xi>=\frac{1}{2}\left[\left(A_{\sigma}^{\left[i_{1} i_{2}\right]}-\frac{2}{3} d_{a} A_{\sigma}^{\left[i_{1} i_{2}\right] a}\right) \Xi^{\sigma}+\frac{4}{3} A_{\sigma}^{\left[i_{1} i_{2}\right] j} \Xi_{j}^{\sigma}\right] \wedge d s_{i_{1} i_{2}} .
$$

it is immediate to get:

$\alpha:=\mathbb{T}^{\prime}-\mathbb{T}=-\frac{1}{6}\left[d_{a} A_{\sigma}^{\left[i_{1} i_{2}\right] a} \omega^{\sigma}-A_{\sigma}^{\left[i_{1} i_{2}\right] a} \omega_{a}^{\sigma}\right] \wedge d s_{i_{1} i_{2}}=-\frac{1}{6} d_{a}\left(A_{\sigma}^{\left[i_{1} i_{2}\right] a} \omega^{\sigma}\right) \wedge d s_{i_{1} i_{2}}$. 
We note that $\alpha$ can be put in the form of a local divergence and in agreement with Lemma 4.1 one can check that indeed $\mathcal{D} \alpha \equiv 0$.

Therefore we understand that the difference among the variational morphism splitting as stated in coordinates in [3] and our "canonical splitting"like decomposition consists in passing from one splitting to the other one just by adding and substracting an identically vanishing divergence, an aspect which deals with the local nature of our decomposition.

As we shall see, the relation (25) suggests a sort of 'canonical' construction of Lepage equivalents other than the usual Poincaré-Cartan form (see also the discussion about this point in [16]). The uniqueness and globality properties of the terms $\mathbb{E}^{\prime}$ and $\mathbb{T}^{\prime}$ remain to be deeper investigated; in particular, the difference $\mathbb{T}^{\prime}-\mathbb{T}$ is a closed form, and, if global, it defines a de Rham cohomology class. This feature deals with other topological aspects of Lagrangian field theories [2, 5, 6, 15, 17] which will be the subject of future research.

\section{The Krupka-Betounes equivalent for first order theories and a first glance to the sec- ond order}

Suppose we have a fibered manifold $\pi: Y \longrightarrow X$, with $\operatorname{dim} X=n$ and $\operatorname{dim} Y=n+m$. Consider a local chart $\left(x^{i}, y^{\sigma}\right), 1 \leq i \leq n, 1 \leq \sigma \leq m$ on $Y$, then on $J^{1} Y$ we have local fibered coordinates $\left(x^{i}, y^{\sigma}, y_{j}^{\sigma}\right)$, with $1 \leq i, j \leq n$ and $1 \leq \sigma \leq m$.

Let us consider a Lagrangian $\lambda$, defined on $J^{1} Y$, locally expressed as $\lambda=\mathscr{L}\left(x^{i}, y^{\sigma}, y_{j}^{\sigma}\right) d s$. As already mentioned, in [16] (Lemma 4.5, example 4.6) a Residual operator for lower degree forms was obtained by the first author for the case $s=1$. Olga Rossi [18] conjectured that, making use of it, the Krupka-Betounes Lepage equivalent for first order theories could be obtained by the application of the following recurrence formulae:

$$
\begin{aligned}
& \rho_{1}=\lambda-p_{1} \mathcal{R}(d \lambda)=\theta_{\lambda} \quad \text { (Poincaré-Cartan form of the Lagrangian) } \\
& \rho_{2}=\rho_{1}-p_{2} \mathscr{R}\left(d \rho_{1}\right) \\
& \rho_{3}=\rho_{2}-p_{3} \mathscr{R}\left(d \rho_{2}\right) \\
& \ldots \\
& \rho_{n}=\rho_{n-1}-p_{n} \mathscr{R}\left(d \rho_{n-1}\right)
\end{aligned}
$$


with $\mathcal{R}$ and $\mathscr{R}$ the residual operator for top forms and lower degree forms, respectively.

In the following, in view of a generalization to the second order case, we apply the recurrence formulae by using the characterization of the Residual operator for lower degree forms as given in the present paper by Proposition 4.1 .

We compute explicitly the forms $\rho_{1}, \ldots, \rho_{n}$. First we have the well-known Poincaré-Cartan form of $\lambda$ :

$$
\rho_{1}=\theta_{\lambda}=\mathscr{L} d s+p_{\sigma}^{i} \omega^{\sigma} \wedge d s_{i} \quad p_{\sigma}^{i}:=\frac{\partial \mathscr{L}}{\partial y_{i}^{\sigma}} .
$$

In order to compute $\rho_{2}$ we observe that the Residual operator $\mathscr{R}$ does not change the order of contactness of its argument, i.e. $p_{2} \mathscr{R}\left(d \rho_{1}\right)=\mathscr{R}\left(p_{2} d \rho_{1}\right)$. Thus we can reduce ourselves to consider only the 2-contact component of the differential of $\rho_{1}$. In particular:

$$
\begin{aligned}
& p_{2} d \rho_{1}=\omega^{\sigma} \wedge\left(\partial_{\sigma} p_{\nu}^{i} \omega^{\nu} \wedge d s_{i}\right)+\omega_{j}^{\sigma} \wedge\left(\partial_{\sigma}^{j} p_{\nu}^{i} \omega^{\nu} \wedge d s_{i}\right)= \\
& =\omega^{\sigma} \wedge\left[\partial_{\sigma} p_{\nu}^{i} \omega^{\nu} \wedge d s_{i}-d_{j}\left(\partial_{\sigma}^{j} p_{\nu}^{i} \omega^{\nu} \wedge d s_{i}\right)\right]+d_{j}\left[\omega^{\sigma} \wedge\left(\partial_{\sigma}^{j} p_{\nu}^{i} \omega^{\nu} \wedge d s_{i}\right)\right]
\end{aligned}
$$

Specializing Proposition 4.1 we have:

$$
\mathscr{R}\left(p_{2} d \rho_{1}\right)=\frac{1}{2} \partial_{\sigma}^{j} p_{\nu}^{i} \omega^{\sigma} \wedge \omega^{\nu} \wedge d s_{i j} .
$$

Thus

$$
\rho_{2}=\rho_{1}-p_{2} \mathscr{R}\left(d \rho_{1}\right)=\mathscr{L} d s+p_{\sigma}^{i} \omega^{\sigma} \wedge d s_{i}+\frac{1}{2} \partial_{\sigma}^{i} p_{\nu}^{j} \omega^{\sigma} \wedge \omega^{\nu} \wedge d s_{i j} .
$$

Now we compute $\rho_{3}=\rho_{2}-p_{3} \mathscr{R}\left(d \rho_{2}\right)$. Again we can restrict our attention to the term

$$
\begin{aligned}
p_{3} d \rho_{2}=\frac{1}{2}\left(\partial_{\sigma_{1}} \partial_{\sigma_{2}}^{i} p_{\sigma_{3}}^{j} \omega^{\sigma_{1}} \wedge \omega^{\sigma_{2}} \wedge \omega^{\sigma_{3}}\right. & \left.\wedge d s_{i j}+\partial_{\sigma_{1}}^{k} \partial_{\sigma_{2}}^{i} p_{\sigma_{3}}^{j} \omega_{k}^{\sigma_{1}} \wedge \omega^{\sigma_{2}} \wedge \omega^{\sigma_{3}} \wedge d s_{i j}\right) \\
=\omega^{\sigma_{1}} \wedge(\ldots)+d_{k}\left[\omega^{\sigma_{1}}\right. & \left.\wedge \frac{1}{2}\left(\partial_{\sigma_{1}}^{k} \partial_{\sigma_{2}}^{i} p_{\sigma_{3}}^{j} \omega^{\sigma_{2}} \wedge \omega^{\sigma_{3}} \wedge d s_{i j}\right)\right]
\end{aligned}
$$

Thence

$$
p_{3} \mathscr{R}\left(d \rho_{2}\right)=-\frac{1}{6} \partial_{\sigma_{1}}^{k} \partial_{\sigma_{2}}^{i} p_{\sigma_{3}}^{j} \omega^{\sigma_{1}} \wedge \omega^{\sigma_{2}} \wedge \omega^{\sigma_{3}} \wedge d s_{i j k}
$$


and finally

$$
\begin{aligned}
& \rho_{3}=\rho_{2}-p_{3} \mathscr{R}\left(d \rho_{2}\right)=\mathscr{L} d s+p_{\sigma}^{i} \omega^{\sigma} \wedge d s_{i}+\frac{1}{2 !} \partial_{\sigma_{1}}^{i_{1}} p_{\sigma_{2}}^{i_{2}} \omega^{\sigma_{1}} \wedge \omega^{\sigma_{2}} \wedge d s_{i_{1} i_{2}}+ \\
& +\frac{1}{3 !} \partial_{\sigma_{1}}^{i_{1}} \partial_{\sigma_{2}}^{i_{2}} p_{\sigma_{3}}^{i_{3}} \omega^{\sigma_{1}} \wedge \omega^{\sigma_{2}} \wedge \omega^{\sigma_{3}} \wedge d s_{i_{1} i_{2} i_{3}} .
\end{aligned}
$$

Proceeding in this way it is straightforward to see that

$$
\rho_{n}=\mathscr{L} d s+\sum_{q=1}^{n} \frac{1}{q !} \frac{\partial^{q} \mathscr{L}}{\partial y_{i_{1}}^{\sigma_{1}} \ldots \partial y_{i_{q}}^{\sigma_{q}}} \omega^{\sigma_{1}} \wedge \cdots \wedge \omega^{\sigma_{q}} \wedge d s_{i_{1} \ldots i_{q}}
$$

which is known as the Krupka-Betounes equivalent of the Lagrangian $\lambda[1$, 10, 11, 19, 21].

Example 5.1. We use the notation adopted in Example 3.5 of article [16]. The $(1+1)$-dimensional free quantum particle is a first order theory for the fibered manifold $\pi: \mathbb{R}^{2} \times \mathbb{R}^{2} \rightarrow \mathbb{R}^{2}$, with coordinates $(t, x, v, w)$, described by the Lagrangian

$$
\lambda=-\left(\frac{\hbar^{2}}{4 m}\left(v_{x}^{2}+w_{x}^{2}\right)+\frac{\hbar}{2}\left(v w_{t}-v_{t} w\right)\right) d t \wedge d x=\mathscr{L}\left(j^{1} \pi\right) d t \wedge d x .
$$

In order to compute $\rho_{1}=\lambda-p_{1} \mathcal{R}(d \lambda)$, we first have to consider $p_{1} \mathcal{R}(d \lambda)=$ $\mathcal{R}\left(p_{1} d \lambda\right)$. We have:

$p_{1} d \lambda=\left(-\frac{\hbar}{2} w_{t} \omega^{1}+\frac{\hbar}{2} v_{t} \omega^{2}+\frac{\hbar}{2} w \omega_{t}^{1}-\frac{\hbar}{2} v \omega_{t}^{2}-\frac{\hbar^{2}}{2 m} v_{x} \omega_{x}^{1}-\frac{\hbar^{2}}{2 m} w_{x} \omega_{x}^{2}\right) d t \wedge d x$.

Then

$p_{1} \mathcal{R}(d \lambda)=\mathcal{R}\left(p_{1} d \lambda\right)=-\frac{\hbar}{2}\left(w \omega^{1} \wedge d x-v \omega^{2} \wedge d x+\frac{\hbar}{m} v_{x} \omega^{1} \wedge d t+\frac{\hbar}{m} w_{x} \omega^{2} \wedge d t\right)$

and finally

$$
\rho_{1}=\lambda+\frac{\hbar}{2}\left(w \omega^{1} \wedge d x-v \omega^{2} \wedge d x+\frac{\hbar}{m} v_{x} \omega^{1} \wedge d t+\frac{\hbar}{m} w_{x} \omega^{2} \wedge d t\right) .
$$

Now the following (and last) step is $\rho_{2}=\rho_{1}-p_{2} \mathscr{R}\left(d \rho_{1}\right)$. We have:

$p_{2} d \rho_{1}=\frac{\hbar}{2}\left(\omega^{2} \wedge \omega^{1} \wedge d x-\omega^{1} \wedge \omega^{2} \wedge d x+\frac{\hbar}{m} \omega_{x}^{1} \wedge \omega^{1} \wedge d t+\frac{\hbar}{m} \omega_{x}^{2} \wedge \omega^{2} \wedge d t\right)$.

But then $p_{2} \mathscr{R}\left(d \rho_{1}\right)=\mathscr{R}\left(p_{2} d \rho_{1}\right)=0$, i.e. $\rho_{2}=\rho_{1}$ is just the Poincaré-Cartan form. This is due to the specific form of the Lagrangian, indeed the only non zero second partial derivatives of $\mathscr{L}\left(j^{1} \pi\right)$ contributes to (27) within vanishing terms involving double wedge products of same $\omega$ 's. 
Geometric integration by parts and Lepage equivalents

\subsection{Lepage equivalents for second order theories}

Let us now consider a second order Lagrangian $\lambda=\mathscr{L}\left(x^{i}, y^{\sigma}, y_{j}^{\sigma}, y_{j k}^{\sigma}\right) d s$. As a first step we compute the Poincaré-Cartan form of the Lagrangian using the first of Olga Rossi's recurrence formulae: $\rho_{1}=\lambda-p_{1} \mathcal{R}(d \lambda)=\theta_{\lambda}$.

$$
\begin{aligned}
& d \lambda=p_{\sigma} \omega^{\sigma} \wedge d s+p_{\sigma}^{j} \omega_{j}^{\sigma} \wedge d s+p_{\sigma}^{j k} \omega_{j k}^{\sigma} \wedge d s= \\
& =d_{j} d_{k}\left(p_{\sigma}^{j k} \omega^{\sigma} \wedge d s\right)+d_{j}\left[\left(p_{\sigma}^{j}-2 d_{k} p_{\sigma}^{j k}\right) \omega^{\sigma} \wedge d s\right]+\left(d_{k} d_{j} p_{\sigma}^{j k}-d_{j} p_{\sigma}^{j}+p_{\sigma}\right) \omega^{\sigma} \wedge d s .
\end{aligned}
$$

Using the formula for the residual operator in this particular case:

$$
\mathcal{R}=\sum_{|I|=0}^{1}(-1)^{1} d_{I} \chi^{I j} \wedge d s_{j},
$$

we obtain:

$$
p_{1} \mathcal{R}(d \lambda)=-\left[\left(p_{\sigma}^{j}-2 d_{k} p_{\sigma}^{j k}+d_{k} p_{\sigma}^{j k}\right) \omega^{\sigma} \wedge d s_{j}+p_{\sigma}^{j k} \omega_{k}^{\sigma} \wedge d s_{j}\right] .
$$

Then, as expected, the Poincaré-Cartan form of the Lagrangian $\lambda$ is:

$$
\theta_{\lambda}=\mathscr{L} d s+\left(p_{\sigma}^{j}-d_{k} p_{\sigma}^{j k}\right) \omega^{\sigma} \wedge d s_{j}+p_{\sigma}^{j k} \omega_{k}^{\sigma} \wedge d s_{j}
$$

We now rename $f_{\sigma}^{j}:=p_{\sigma}^{j}-d_{k} p_{\sigma}^{j k}$ and $f_{\sigma}^{j k}:=p_{\sigma}^{j k}$ and continue computing the second of Rossi's recurrence formulae: $\rho_{2}=\theta_{\lambda}-p_{2} \mathscr{R}\left(d \theta_{\lambda}\right)$.

We are interested only in the 2 -contact component of $d \theta_{\lambda}$ because $p_{2} \mathscr{R}=$ $p_{2} \mathscr{R} p_{2}$, i.e. the Residual operator $\mathscr{R}$ does not increase the order of contactness of its argument, thus let us write

$$
\begin{aligned}
& p_{2}\left(d \theta_{\lambda}\right)=\omega^{\sigma} \wedge\left(\partial_{\sigma} f_{\sigma_{2}}^{i} \omega^{\sigma_{2}} \wedge d s_{i}-\partial_{\sigma_{1}}^{j_{1}} f_{\sigma}^{i} \omega_{j_{1}}^{\sigma_{1}} \wedge d s_{i}-\partial_{\sigma_{1}}^{j_{1} j_{2}} f_{\sigma}^{i} \omega_{j_{1} j_{2}}^{\sigma_{1}} \wedge d s_{i}+\right. \\
& \left.+\partial_{\sigma} f_{\sigma_{2}}^{i j_{2}} \omega_{j_{2}}^{\sigma_{2}} \wedge d s_{i}\right)+\omega_{j}^{\sigma} \wedge\left(\partial_{\sigma}^{j} f_{\sigma_{2}}^{i j_{2}} \omega_{j_{2}}^{\sigma_{2}} \wedge d s_{i}-\partial_{\sigma_{1}}^{j_{1} j_{3}} f_{\sigma}^{i j} \omega_{j_{1} j_{3}}^{\sigma_{1}} \wedge d s_{i}\right) .
\end{aligned}
$$

Now we recast this expression in the form $\sum_{|I|=0}^{1} d_{I}\left(\omega^{\sigma} \wedge \xi_{\sigma}^{I}\right)$ and apply the integration by parts lemma, so to get for the Residual operator

$$
\begin{aligned}
& p_{2} \mathscr{R}\left(d \theta_{\lambda}\right)=\frac{1}{2} \partial_{\sigma}^{j} f_{\sigma_{2}}^{i j_{2}} \omega_{j_{2}}^{\sigma_{2}} \wedge d s_{i j}-\frac{1}{2} \partial_{\sigma_{1}}^{j_{1} j_{3}} f_{\sigma}^{i j} \omega_{j_{1} j_{3}}^{\sigma_{1}} \wedge d s_{i j}= \\
& =\frac{1}{2} \partial_{\sigma}^{j} f_{\sigma_{2}}^{i j_{2}} \omega_{j_{2}}^{\sigma_{2}} \wedge d s_{i j}
\end{aligned}
$$

being $f_{\sigma}^{i j}$ symmetric in $(i j)$. Hence we obtain the following formula for $\rho_{2}$ :

$$
\rho_{2}=\mathscr{L} d s+f_{\sigma}^{i} \omega^{\sigma} \wedge d s_{i}+f_{\sigma}^{i j} \omega_{j}^{\sigma} \wedge d s_{i}+\frac{1}{2} \partial_{\sigma_{1}}^{i_{1}} f_{\sigma_{2}}^{i_{2} j_{2}} \omega^{\sigma_{1}} \wedge \omega_{j_{2}}^{\sigma_{2}} \wedge d s_{i_{1} i_{2}}
$$


It turns out that it is possible to apply this reasoning at any step of Rossi's recurrence formulae, e.g. at the third step we get

$$
p_{3} \mathscr{R}\left(d \rho_{2}\right)=-\frac{1}{6} \partial_{\sigma_{1}}^{i_{1}} \partial_{\sigma_{2}}^{i_{2}} f_{\sigma}^{i_{3} j} \omega^{\sigma_{1}} \wedge \omega^{\sigma_{2}} \wedge \omega_{j}^{\sigma} \wedge d s_{i_{1} i_{2} i_{3}} .
$$

Proceeding in this way, the final expression of $\rho_{n}$ has the form:

$$
\begin{aligned}
& \rho_{n}=\mathscr{L} d s+f_{\sigma}^{i} \omega^{\sigma} \wedge d s_{i}+ \\
& +\sum_{q=1}^{n} \frac{1}{q !} \frac{\partial \mathscr{L}}{\partial y_{i_{1}}^{\sigma_{1}} \ldots \partial y_{i_{q-1}}^{\sigma_{q-1}} \partial y_{i_{q} j}^{\sigma_{q}}} \omega^{\sigma_{1}} \wedge \ldots \wedge \omega^{\sigma_{q-1}} \wedge \omega_{j}^{\sigma_{q}} \wedge d s_{i_{1} \ldots i_{q}}
\end{aligned}
$$

We remark that the integration by parts is not uniquely defined in the case $r=2$. Indeed, if we take into account in the decomposition of $p_{2}\left(d \rho_{1}\right)=$ $p_{2}\left(d \theta_{\lambda}\right)$ also the term $\omega_{j}^{\sigma} \wedge\left(\partial_{\sigma}^{j} f_{\sigma_{2}}^{i} \omega^{\sigma_{2}} \wedge d s_{i}\right)$ and work on it as above, we get an additional term in $\rho_{n}$, thus obtaining a sort of generalization at the second order of the Krupka-Betounes equivalent, namely :

$$
\begin{gathered}
\rho_{n}=\mathscr{L} d s+\sum_{q=1}^{n} \frac{1}{q !} \frac{\partial \mathscr{L}}{\partial y_{i_{1}}^{\sigma_{1}} \ldots \partial y_{i_{q-1}}^{\sigma_{q-1}} \partial y_{i_{q} j}^{\sigma_{q}}} \omega^{\sigma_{1}} \wedge \ldots \wedge \omega^{\sigma_{q-1}} \wedge \omega_{j}^{\sigma_{q}} \wedge d s_{i_{1} \ldots i_{q}}+ \\
+f_{\sigma}^{i} \omega^{\sigma} \wedge d s_{i}+\sum_{q=1}^{n-1} \frac{1}{(q+1) !} \frac{\partial f_{\sigma_{q+1}}^{i_{q+1}}}{\partial y_{i_{1}}^{\sigma_{1}} \ldots \partial y_{i_{q}}^{\sigma_{q}}} \omega^{\sigma_{1}} \wedge \ldots \wedge \omega^{\sigma_{q}} \wedge \omega^{\sigma_{q+1}} \wedge d s_{i_{1} \ldots i_{q} i_{q+1}},
\end{gathered}
$$

which exactly reduces to the Krupka-Betounes equivalent (27), when the Lagrangian is of order $r=1$ (see [14] for a review on Lepage equivalents of order $r \geq 1$ ).

The study of the properties of this Lepage equivalent according to [1] (see also [22]) will be the subject of a separate paper.

Remark 5.1. We point out that Rossi's recurrence formulae provide a well defined second order Lepage equivalent $\rho_{n}$, satisfying the well known defining properties:

- $h \rho_{n}=\lambda$

- $\mathcal{E}_{\lambda}=p_{1} d \rho_{n}$ is a source form.

Indeed, the first condition is trivially verified since all the terms that are added to the Lagrangian in the recurrence steps are contact and thence do not affect the horizontal part of $\rho_{n}$. 
Furthermore, we note that the only terms in $\rho_{n}$ that can contribute to the 1-contact component of the differential are the horizontal terms (they contribute via the contact differential $d_{C}$ ) and the 1-contact terms (they contribute via the horizontal differential $d_{H}$ ). In other words, we have $p_{1} d \rho_{n}=p_{1} d \rho_{1}$.

Now, $\rho_{1}$ is a distinguished Lepage equivalent, the so-called principal Lepage equivalent; hence $p_{1} d \rho_{1}$ is by definition a source form and this shows that $\rho_{n}$ is indeed a Lepage equivalent too. The proof for any order $r$ can be done in analogy with [16], Theorem 3.11.

\section{Acknowledgements}

The first author (MP) was supported by the Department of Mathematics University of Torino project PALM_RILO_20_01 and would like to acknowledge the contribution of the COST Action CA17139. The third author (FZ) was also supported by a $\mathrm{PhD}$ grant of the University of Göttingen.

\section{References}

[1] D.E. Betounes: Extension of the classical Cartan form, Phys. Rev. D29 (1984) 599.

[2] F. Cattafi, M. Palese, E. Winterroth: Variational derivatives in locally Lagrangian field theories and Noether-Bessel-Hagen currents, Int. Journ. Geom. Meth. Mod. Phys 13 (8) (2016)1650067.

[3] L. Fatibene, M. Francaviglia: Natural and Gauge Natural Formalism for Classical Field Theories, Springer (2003).

[4] M. Ferraris: Fibered connections and global Poincaré-Cartan forms in higher-order calculus of variations, Proc. Conf. Diff. Geom. Appl., Part 2, Univ. J. E. Purkyně Brno (1984) 61-91.

[5] M. Ferraris, M. Palese, E. Winterroth: Local variational problems and conservation laws, Diff. Geom. Appl. 29 (1) (2011) S80-S85.

[6] M. Francaviglia, M. Palese, E. Winterroth: Variationally equivalent problems and variations of noether currents Int. Journ. Geom. Meth. Mod. Phys 10 (1) (2013) 1220024. 
Geometric integration by parts and Lepage equivalents

[7] M. Horák, I. Kolár: On the higher order Poincaré-Cartan forms, Czechoslovak Math. J., 33 (108) (1983), 467-475.

[8] I. Koláŕ, P.W. Michor, J. Slovák: Natural Operations in Differential Geometry, Springer-Verlag, New York, 1993 USA.

[9] M. Krbek, J. Musilová: Representation of the Variational Sequence by Differential Forms, Acta Appl. Math. 88 (2) (2005) 177-199.

[10] D. Krupka, Some Geometric Aspects of Variational Problems in Fibered Manifolds, Folia Fac. Sci. Nat. UJEP Brunensis, Physica 14,(1973) 65 pp.; see also arXiv:math-ph/0110005.

[11] D. Krupka: A map associated to the Lepagean forms of the calculus of variations in fibered manifolds, Czech. Math. J. 27 (1977) 114-118.

[12] D. Krupka: Variational Sequences on Finite Order Jet Spaces, in: J. Janyška and D. Krupka (eds), Proc. Conf. Diff. Geom. Appl., Brno, Czechoslovakia, 1989, World Scientific, Singapore, (1990) 236-254.

[13] D. Krupka: Introduction to Global Variational Geometry, Atlantis Studies in Variational Geometry, Atlantis Press, Paris (2015).

[14] O. Krupková: Lepage forms in the calculus of variations, in Variations, Geometry and Physics, O. Krupková, D. Saunders eds., Nova Science Publishers, Inc. New York (2009).

[15] M. Palese: Variations by generalized symmetries of local Noether strong currents equivalent to global canonical Noether currents, Comm. Math. 24 (2) (2016) pp. 125-135; Erratum, Comm. Math. 25 (1) (2017) 71-72.

[16] M. Palese, O. Rossi, E. Winterroth, J. Musilová: Variational Sequences, Representation Sequences and Applications in Physics, SIGMA 12 (2016) N. 045, 45 pp.

[17] M. Palese, E. Winterroth: Topological obstructions in Lagrangian field theories, with an application to 3D Chern-Simons gauge theory, Jour. Math. Phys. 58(2) (2017) 023502.

[18] O. Rossi: private communication to the first author (MP). 
Geometric integration by parts and Lepage equivalents

[19] J. Pérez Álvarez: On the Cartan-Betounes form, Mathematische Nachrichten 292 (8) (2019) 1-9.

[20] D.J. Saunders: The Geometry of Jet Bundles, Cambridge University Press, Cambridge, UK (1989).

[21] D. Saunders: Some geometric aspects of the calculus of variations in several independent variables, Comm. Math. 18 (1) (2010) 3-19.

[22] N. Voicu, S. Garoiu, B. Vasian: On the closure property of Lepage equivalents of Lagrangians, preprint arXiv:2102.12955. 